\title{
Orally Deliverable Dual-Targeted Pellets for the Synergistic Treatment of Ulcerative Colitis
}

\author{
Xiaomeng Tang ${ }^{1-3, *}$ \\ Meng Yang ${ }^{4, *}$ \\ Yongwei $\mathrm{Gu}^{1,2}$ \\ Liangdi Jiang ${ }^{1,2,5}$ \\ Yue $\mathrm{Du}^{1,2,5}$ \\ Jiyong Liu ${ }^{1-3}$
}

'Department of Pharmacy, Fudan University Shanghai Cancer Center, Fudan University, Shanghai, 200032, People's Republic of China; ${ }^{2}$ Department of Oncology, Shanghai Medical College, Fudan University, Shanghai, 200032, People's Republic of China; ${ }^{3}$ Department of Pharmacy, Changhai Hospital, Naval Medical University, Shanghai, 200433, People's Republic of China; ${ }^{4}$ Department of Pharmacy, Shanghai Ninth People Hospital, Shanghai Jiao Tong University, Shanghai, 2000II, People's Republic of China; ${ }^{5}$ College of Pharmacy, Shandong University of Traditional Chinese Medicine, Jinan, Shandong, 250355, People's Republic of China

*These authors contributed equally to this work

\begin{abstract}
Purpose: The effective treatment of ulcerative colitis (UC) poses substantial challenges, and the aetiopathogenesis of UC is closely related to infectious, immunological and environmental factors. Currently, there is a considerable need for the development of orally bioavailable dosage forms that enable the effective delivery of therapeutic drugs to local diseased lesions in the gastrointestinal tract.
\end{abstract}

Methods: Berberine (BBR) and Atractylodes macrocephala Koidz (AM) volatile oil, derived from the Chinese herbs Coptis chinensis Franch and Atractylodes macrocephala Koidz, have anti-inflammatory and immunomodulatory activities. In this study, we prepared colon-targeted pellets loaded with BBR and stomach-targeted pellets loaded with AM volatile oil for the synergistic treatment of UC. The Box-Behnken design and $\beta$ cyclodextrin inclusion technique were used to optimize the enteric coating formula and prepare volatile oil inclusion compounds.

Results: The two types of pellets were spherical and had satisfactory physical properties. The pharmacokinetic results showed that the AUC and MRT values of the dual-targeted (DPs) pellets were higher than those of the control pellets. In addition, in vivo animal imaging confirmed that the DPs could effectively deliver BBR to the colon. Moreover, compared with sulfasalazine and monotherapy, DPs exerted a more significant antiinflammatory effect by inhibiting the expression of inflammatory factors including IL-1 $\beta$, IL-4, IL-6, TNF- $\alpha$ and MPO both in serum and tissues and enhancing immunity by decreasing the production of IgA and IgG.

Conclusion: The DPs play a synergistic anti-UC effect by exerting systemic and local antiinflammatory and provide an effective oral targeted preparation for the treatment of UC.

Keywords: ulcerative colitis, oral dual-targeted pellets, synergistic treatment, berberine, AM volatile oil

\section{Introduction}

Ulcerative colitis (UC), known as chronic nonspecific ulcerative colitis, is a chronic and debilitating inflammatory disease that results from a complex interplay between infectious, immunological, environmental and microbial factors. ${ }^{1}$ The main clinical manifestations of UC are digestive system symptoms and difficulty healing, for as long as several months to decades. ${ }^{2,3}$ Depending on the duration and extent of inflammation, long-standing UC patients $(\sim 18 \%)$ have a much higher risk of developing colitis-associated colorectal cancer (CAC). ${ }^{4-6}$ At present, adrenal corticosteroids (prednisone) and aminosalicylic acid (mesalazine) are commonly used drugs for the treatment of UC, but these drugs can only relieve symptoms and often have many side effects. In addition, the
Correspondence: Jiyong Liu

University Shanghai Cancer Center, Fudan

University, Shanghai, 200032, People's

Republic of China

Tel/Fax +86-2I-64I75590

Email liujiyong@fudan.edu.cn 


\section{Graphical Abstract}

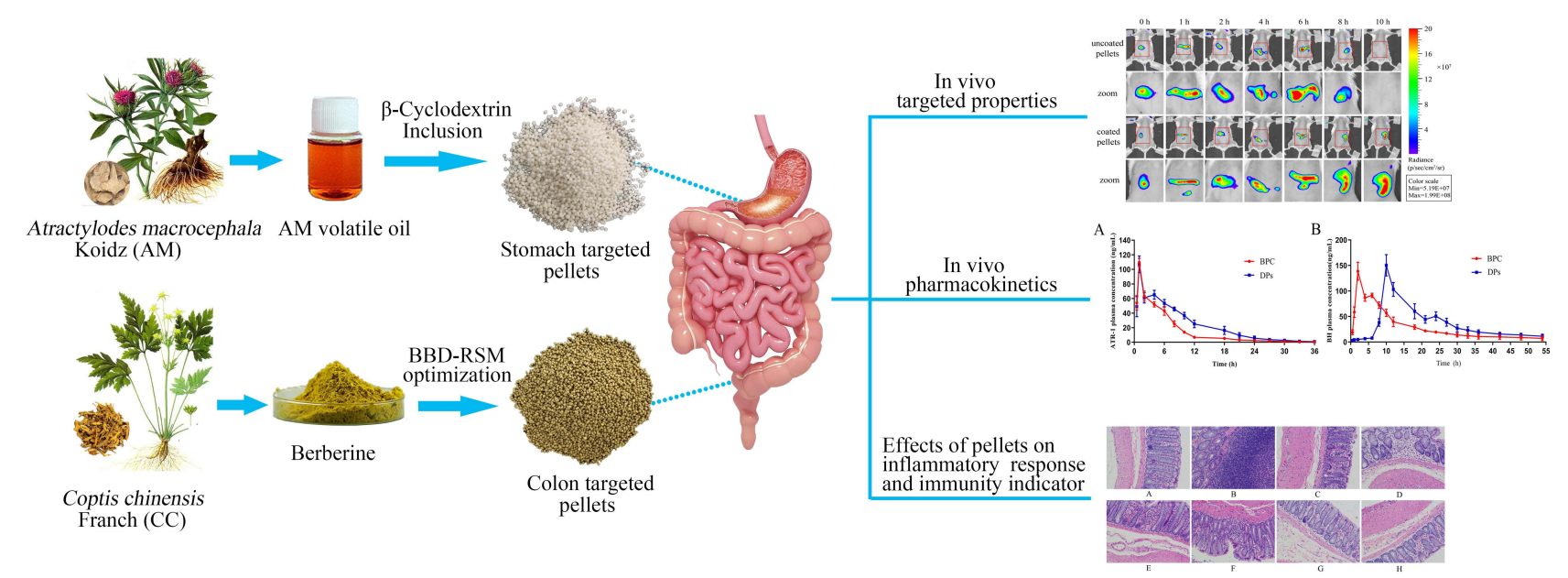

effectiveness and safety of other reported drugs, such as immunosuppressive agents (azathioprine), vary greatly among different individuals. ${ }^{7}$ Therefore, there is a considerable need to develop expedient and practical formulations that can effectively treat UC.

Atractylodes macrocephala Koidz (AM) has long been used to treat gastrointestinal hypofunction. ${ }^{8}$ Volatile oil contains volatile components extracted from $\mathrm{AM}$ and can improve gastrointestinal function, enhance body immunity and exert anti-inflammatory effects. ${ }^{9}$ However, the components of AM volatile oil are less stable and can easily decompose and deteriorate under the action of oxygen, light and heat. As reported, the technique of cyclodextrin inclusion can prevent oxidation and decomposition of the volatile oil and simultaneously make the liquid drug a powder, which can improve the drug stability during the preparation and storage processes. ${ }^{10}$ Further pelleting of the inclusion complex can facilitate patient administration and mask undesirable odours of the volatile oil.

Berberine (BBR) is the active ingredient of Coptis chinensis Franch (CC) and can reduce the symptoms of colitis, attenuate mucosal barrier damage, ${ }^{11}$ and restore barrier function. ${ }^{12}$ However, BBR is a BCS class II compound that exhibits low bioavailability when orally administered and is mainly well absorbed in the intestine. ${ }^{13}$ Therefore, it is necessary to improve the solubility and bioavailability of BBR and to facilitate targeted delivery of BBR to diseased colon tissues to enhance the local antiinflammatory effect.
Dosage forms play an important role in the absorption and transport of oral preparations. However, clinical treatments for UC are always absorbed or degraded before reaching the colon, leading to less accumulation in ulcer lesions and affecting efficacy. The oral colon-targeted drug delivery system (OCDDS) uses appropriate preparation technology to prevent the drug from being released in the stomach, duodenum, jejunum and front end of the ileum after oral administration. ${ }^{14,15}$ Thus, it can deliver drugs to the lesion and exert a local or systemic therapeutic effect. ${ }^{16}$ There are various types of oral colon-targeted drug delivery systems, including $\mathrm{pH}$ type, enzymatic contact type, time-dependent type, pressure control type, bioadhesive type, prodrug type, combination type, etc. $^{17,18}$ At present, the varieties that have been on the market or entered clinical research are mostly coated with pH-dependent materials. In addition, pellets are a multiunit drug delivery system that can increase the contact area between the drug and gastrointestinal tract, promoting absorption and bioavailability. ${ }^{19}$ Therefore, the development of oral colon-targeted pellets may improve BBR oral bioavailability and colon targeting.

Currently, commercially available OCDDS are mostly of the pH-sensitive coating type based on the $\mathrm{pH}$ of the gastrointestinal tract (stomach 0.9-1.5, small intestine 6.57, colon $6.8-7.5) .{ }^{20}$ Directly marketed polymer materials, such as EUDRAGIT ${ }^{\circledR} \mathrm{L}$ 30D-55 and EUDRAGIT ${ }^{\circledR} \mathrm{FS}$ $30 \mathrm{D}$, with intrinsic $\mathrm{pH}$-sensitive properties, are not suitable for targeted drug delivery to the colon. ${ }^{21}$ Therefore, we optimized mixed polymer materials to allow the coated 
preparation to release a large amount of the drug after reaching the colon.

In this study, BBR and AM volatile oils were loaded into colon-targeted pellets and stomach-targeted pellets, respectively, to prepare DPs for synergistic treatment of UC. After administration, BBR was released in the colon to exert a local targeting anti-inflammatory effect, while $\mathrm{AM}$ volatile oil was absorbed in the stomach to exert a systemic immunoregulatory effect. In this study, we optimized the pellet preparation process, characterized the pellet physicochemical properties and evaluated colonic targeting. In addition, the pharmacokinetic characteristics of DPs in rats were detected. A DSS-induced rat UC model was constructed to investigate the pharmacodynamic properties of the developed DPs. These results could substantially contribute to UC management and might provide a pharmaceutical strategy for the treatment of gastrointestinal diseases.

\section{Materials and Methods Materials}

Atractylodes macrocephala Koidz volatile oil and Coptis chinensis Franch extract were purchased from Xi'an Xiaocao Plant Technology Co., Ltd. (Xi'an, Shanxi, China). Atractylenolide I (AT-1) and berberine hydrochloride standards were purchased from the National Institute for Food and Drug Control (Beijing, China). $\beta$ Cyclodextrin $(\beta-\mathrm{CD})$ was purchased from Tianli Pharmaceutical Excipients Co., Ltd. (Qufu, Shandong, China). Microcrystalline cellulose (MCC) was purchased from Huzhou Linghu Xinwang Chemical Co., Ltd. (Huzhou, Zhejiang, China). Lactose was purchased from DMV-Fonterra Excipients GmbH \& Co. KG (Goch, Germany). Crosslinking polyvinylpyrrolidone XL was purchased from Shanghai Yuanye Biological Technology Co., Ltd. (Shanghai, China). Tween 80 was purchased from Shanghai Lingfeng Chemical Reagent Co., Ltd. (Shanghai, China). EUDRAGIT ${ }^{\circledR}$ FS 30 D and EUDRAGIT $^{\circledR}$ L 30D-55 were kindly donated by Evonik Röhm GmbH. (Darmstadt, Germany). Hypromellose (HPMC), triethylcitrate (TEC) and sulfasalazine (SASP) were purchased from Aladdin Reagent Co., Ltd. (Shanghai, China). Glycerine monostearate (GMS) was purchased from Alfa Aesar Chemical Co., Ltd. (Ward Hill, US). Dextran sulfate sodium salt (DSS, MV: 36000 5000) was purchased from Shanghai Yisheng Biological Technology Co., Ltd. (Shanghai, China). Sulfo
CY7 NHS ester was purchased from Xi'an Ruixi Biological Technology Co., Ltd. (Xi'an, Shanxi, China). HPLC-grade acetonitrile and methanol were purchased from Tedia Co., Ltd. (Shanghai, China). The other chemicals and solvents used in the study were of analytical reagent grade.

\section{Animals}

Sprague-Dawley (SD) rats (male, $180 \pm 20$ g, Experimental Animal Center of Naval Medical University, Shanghai, China; certificate no. SCXK (Hu) 2017-0002) and BALC/c mice (male, $18 \pm 2 \mathrm{~g}$ ) were utilized for all the in vivo studies. The rats were acclimatized for at least 7 days. Animal experiments were performed according to the protocols evaluated by the ethics committee of the Second Military Medical University (Shanghai, China) and conformed to the National Institutes of Health Guide for the Care and Use of Laboratory Animals.

\section{Preparation of $\beta-C D$ Inclusion Complex and Pellet Cores}

The AM volatile oil/ $\beta-C D$ inclusion complex was first prepared to improve oxidative deterioration while masking the taste. At the same time, the solidification of the liquid drug also laid a foundation for the subsequent preparation of gastric coated pellets. The resulting product was analysed using differential scanning calorimetry (DSC) and X-ray powder diffraction (XRD) to confirm whether the inclusion was successful. The GPs and EPs cores were prepared using the extrusion-spheronization method. EPs cores coated with $4 \%$ HPMC as a sub coating layer (SubPs) were also prepared for further coating. The enteric coating dispersion was prepared by mixing EUDRAGIT $^{\circledR} \mathrm{FS}$ 30D, EUDRAGIT ${ }^{\circledR}$ L 30D-55, GMS, TEC, Tween- 80 and purified water. The coating process parameters are summarized in Table S1. For a more detailed description of the preparation process and characterization parameters, see the Supplementary MaterialMethods.

\section{BBD-RSM-Based Enteric Coating Optimization}

Polymer ratio, plasticizer concentration and coating weight gain are the three key factors that regulate the release rate of film-controlled pellets. Therefore, the range of values of each factor was determined by single-factor experiments in a previous study. Thereafter, a Box-Behnken design (BBD) 
with a three-factor ratio of EUDRAGIT ${ }^{\circledR} \mathrm{FS}$ 30D to EUDRAGIT ${ }^{\circledR} \mathrm{L}$ 30D-55 (g/g, $\left.X_{1}\right)$, plasticizer concentration $\left(\%, X_{2}\right)$ and coating weight gain $\left(\%, X_{3}\right)$ was used to optimize the enteric coating formula. This technology is suitable for the investigation of quadratic response surfaces, thus enabling optimization of a process with a small number of experimental runs. The design consists of replicated centre points and a set of points lying at the midpoint of each edge of the multidimensional cube that defines the area of interest. ${ }^{19}$

Response surface modelling (RSM) was performed with Design Expert 8.0 (StateEase Inc., Minneapolis, $\mathrm{MN})$. Table 1 summarizes the factors and levels of independent variables. The chosen dependent variables were the cumulative release percentage values of BBR dissolved in a determined time (after 2, 6 and $12 \mathrm{~h}$ ).

\section{Characterization of Pellets Morphological Characterization}

Pellet size was represented by the average of the length and breadth values determined using a digital Vernier calliper (Shanghai Meinaite Industry Co., Ltd. Shanghai, China). For each pellet type, 20 pellets were randomly characterized, and an average result was calculated. ${ }^{22}$ The pellet size distribution of GPs and EPs was determined by the sieving method with diameters of 550-880 $\mu \mathrm{m}$ (30-18 mesh) or $830-1400 \mu \mathrm{m}(20-12 \mathrm{mesh})$. The results are shown as the percentage of each size fraction. Pellets coated with a thin gold layer were examined via scanning electron microscopy (SEM; HITACHI-S3400N, Japan) to analyse shape and surface morphology. ${ }^{23}$

\section{Roundness, Friability and Bulk Density}

Plane-critical stability (PCS) was used to reflect the roundness of the pellets. During the measurement, $20 \mathrm{~g}$ of the pellets were laid flat on a glass plate, and one end of the glass plate was slowly lifted. The critical angle $(\theta)$ of the plane formed by the inclined plane and the horizontal plane before the pellets start to roll is measured. The smaller the value of $\theta$ is, the better the roundness of the pellets. ${ }^{19}$
Pellet friability $(F r)$ was determined using a multipurpose tablet tester (78X-3C, Shanghai Huanghai Drug Testing Instrument Co., Ltd. Shanghai, China). Approximately $50 \mathrm{~g}$ of the two pellet types was weighed and added to an abrasion drum together with 200 glass beads ( $2 \mathrm{~mm}$ in diameter). The abrasion drum was rotated at $25 \mathrm{r} / \mathrm{min}$ for $10 \mathrm{~min}$. The content of the abrasion drum was sieved using a $550 \mu \mathrm{m}$ (30 mesh) or $830 \mu \mathrm{m}(20$ mesh) sieve, and the fraction below $550 \mu \mathrm{m}$ or $830 \mu \mathrm{m}$ was weighed. Friability was measured in triplicate and calculated as follows. ${ }^{19}$

$$
\begin{aligned}
& \operatorname{Fr}(\%)=\frac{\text { Fraction }<550 \mu \mathrm{m}(\mathrm{g})}{\text { Total sample }} \times 100(\mathrm{GPs}) \\
& \operatorname{Fr}(\%)=\frac{\text { Fraction }<830 \mu \mathrm{m}(\mathrm{g})}{\text { Total sample }} \times 100(\mathrm{EPs})
\end{aligned}
$$

Bulk density was measured with $50 \mathrm{~g}$ of pellets, which were $(M)$ weighed, placed in a $100 \mathrm{~mL}$ graduated glass cylinder and then dropped from $5 \mathrm{~cm}$ away from the table. ${ }^{24}$ The occupied volume $(V)$ by the pellets was read precisely, and the bulk density (d) was obtained with the formula $\mathrm{d}=M / V$. Three batches of pellets were measured in parallel.

\section{Drug Loading Analysis Using HPLC and HPLC-QQQ-MS}

Drug loading was defined as the percentage of drug embedded in a unit weight of pellets. Fifty milligrams of pellets were ground and dissolved in $50 \mathrm{~mL}$ of methanol. Then, the mixture was ultrasonicated and filtered through a $0.45 \mu \mathrm{m}$ membrane filter. The samples were store at $4{ }^{\circ} \mathrm{C}$ for testing. ${ }^{25}$

For EPs, the BBR concentration was analysed using HPLC (Agilent 1200 series, USA). The mobile phase was composed of acetonitrile and water with $0.05 \mathrm{~mol} / \mathrm{L}$ potassium dihydrogen phosphate (adjusted with phosphoric acid to $\mathrm{pH}=3,30 / 70, \mathrm{v} / \mathrm{v}$ ). The flow rate and wavelength were set at $1 \mathrm{~mL} / \mathrm{min}$ and $345 \mathrm{~nm}$, respectively. For GPs, AT-1 (the active ingredient in AM volatile oil) was

Table I Factors and Their Levels in the Box-Behnken Design and Responses

\begin{tabular}{|l|c|c|c|c|}
\hline \multirow{2}{*}{ Factors } & \multicolumn{2}{|c|}{ Levels } & \multicolumn{2}{c|}{ Responses } \\
\cline { 2 - 4 } & -1 & $\mathbf{0}$ & $\mathrm{I}$ & \\
\hline Ratio of EUDRAGIT ${ }^{\circledR} \mathrm{FS} 30 \mathrm{D}$ to EUDRAGIT ${ }^{\circledR} \mathrm{L} 30 \mathrm{D}-55\left(\mathrm{~g} / \mathrm{g}, X_{1}\right)$ & $1: 2$ & $1.25: 1$ & $2: 1$ & Cumulative drug release in 2 $\mathrm{h}\left(\%, Y_{1}\right)$ \\
Plasticizer concentration $\left(\%, X_{2}\right)$ & 5 & 10 & 15 & Cumulative drug release in 6 $\mathrm{h}\left(\%, Y_{2}\right)$ \\
Coating weight gain $\left(\%, X_{3}\right)$ & 10 & 15 & 20 & Cumulative drug release in $12 \mathrm{~h}\left(\%, Y_{3}\right)$ \\
\hline
\end{tabular}


quantitatively analysed using HPLC-QQQ-MS (Agilent Technologies Inc., USA). The mobile phase was composed of methanol and water with $0.1 \%(\mathrm{v} / \mathrm{v})$ acetic acid $(75 / 25$, $\mathrm{v} / \mathrm{v})$. The other parameters were as follows: column temperature, $35{ }^{\circ} \mathrm{C}$; flow rate, $0.3 \mathrm{~mL} / \mathrm{min}$; dry gas $\left(\mathrm{N}_{2}\right)$ temperature, $350{ }^{\circ} \mathrm{C}$; nebulizer gas $\left(\mathrm{N}_{2}\right)$ pressure, $20 \mathrm{psi}$; fragmentor voltage, $165 \mathrm{eV}$; collision energy, $35 \mathrm{eV}$; positive ion monitoring mode; and $m / z=231.1 \rightarrow 128.1$.

\section{In vitro Drug Release Study}

The $\mathrm{pH}$ dissolution method was used to evaluate drug release from pellets. ${ }^{26}$ The GPs quickly disintegrated completely in the artificial gastric juice, resulting in a high release rate $(\mathrm{pH} 1.2$, cumulative release over $98 \%$ in 5 min). The release of BBR from EPs was determined by using a ChP (2020 edition) dissolution apparatus II (basket method) at a rotation speed of $100 \mathrm{r} / \mathrm{min}$ in $750 \mathrm{~mL}$ of dissolution medium at $37 \pm 0.5{ }^{\circ} \mathrm{C}$ under sink conditions. The dissolution test was performed as follows: $\mathrm{pH} 1.2$ for $2 \mathrm{~h}$ followed by phosphate buffer at $\mathrm{pH} 6.8$ for $4 \mathrm{~h}$ and phosphate buffer at $\mathrm{pH} 7.6$ for $18 \mathrm{~h} .{ }^{27}$ The concentration of BBR in different samples was measured via HPLC after filtration $(0.45 \mu \mathrm{m})$. The dissolution test for Sub-Ps and EPs was carried out six times.

\section{In vivo Imaging-Based Colon-Targeted Study of EPs}

To investigate the transport and release characteristics of EPs in the mouse gastrointestinal tract, the mice were administered fluorescein-containing EPs (during the preparation of the pellet core, CY7 aqueous solution was used instead of water as a wetting agent) or uncoated pellet cores. Then, the mice were anaesthetized with isoflurane and fixed in an imaging apparatus (Quick View 3000, BioReal Sciences Technology Co., Ltd. Austria) at predetermined time points $(0 \mathrm{~h}, 1 \mathrm{~h}, 2 \mathrm{~h}, 4 \mathrm{~h}, 6 \mathrm{~h}, 8 \mathrm{~h}, 10 \mathrm{~h})$ after administration. The excitation light wavelength was set to $655 \mathrm{~nm}$, the emission light wavelength was $716 \mathrm{~nm}$, and the exposure time was $3 \mathrm{~s}^{28}$

\section{In vivo Pharmacokinetics Study}

The rats were fasted for $12 \mathrm{~h}$ with free access to water and were randomly sorted into two groups, with three rats in each group. The two groups were given DPs or bulk pharmaceutical chemicals (BPC, a mixture of BBR and AM volatile oil) by means of oral administration at doses of $200 \mathrm{mg} / \mathrm{kg}$ BBR and $50 \mathrm{mg} / \mathrm{kg}$ AT-1. Blood samples were collected from the rats via the eye retro-orbital vein at specific intervals of $0,0.5,1,2,4,6,8,10,12,18,21$, $24,27,30,33,36,42,48$ and $54 \mathrm{~h}$. The blood samples were immediately subjected to centrifugation (Eppendorf (China), Ltd. Shanghai, China) at $3000 \mathrm{rpm}$ for $15 \mathrm{~min}$. The supernatant was then kept in a freezer at $-20{ }^{\circ} \mathrm{C}$ for further analysis. Before analysis, plasma samples were protein precipitated with methanol and acetonitrile and dried with nitrogen at $40{ }^{\circ} \mathrm{C}$. The sample was reconstituted in $100 \mu \mathrm{L}$ methanol and centrifuged before HPLC-QQQMS analysis.

Standard methods were used to calculate the pharmacokinetic parameters AUC, half-life $\left(t_{1 / 2}\right)$ and MRT using Kinetica 5.0 software (Thermo Fisher Scientific Inc. Waltham, MA, USA). $C_{\max }$ and $T_{\max }$ were directly computed from the plasma concentration versus time plot. Furthermore, the relative bioavailability $\left(F_{\text {rel }}\right)$ with reference to the AUC of the developed DPs group compared to the BPC group was calculated using the equation given below. $^{29}$

$$
F_{r e l} \frac{\mathrm{AUC}^{\mathrm{A}}}{\mathrm{AUC}^{\mathrm{B}}} \times 100 \%
$$

where $F_{\text {rel }}$ is the relative bioavailability and $\mathrm{AUC}^{\mathrm{A}}$ and $\mathrm{AUC}^{\mathrm{B}}$ are the areas under the drug concentration-time curves of DPs (test) and BPC (reference), respectively.

The concentrations of BBR and AT- 1 in plasma were also determined using HPLC-QQQ-MS. The mobile phase was composed of acetonitrile (phase A) and water with $0.1 \%$ (v/v) acetic acid (phase B). For BBR, m/z $=336.2 \rightarrow 320.2$, and the operating parameters were set as follows: nebulizer gas $\left(\mathrm{N}_{2}\right), 20 \mathrm{psi}$; fragmentor voltage, $155 \mathrm{eV}$; collision energy, $30 \mathrm{eV}$; and drying gas $\left(\mathrm{N}_{2}\right)$ temperature, $320{ }^{\circ} \mathrm{C}$. For AT-1, the mobile phase ratio was $\mathrm{A}: \mathrm{B}=75: 25$, and the remaining parameters were the same as before.

\section{Anti-Ulcerative Colitis Analysis Induction of a UC Rat Model and Experimental Design}

UC was induced by replacing the rats' drinking water with a $5 \%(\mathrm{w} / \mathrm{v})$ DSS solution for 7 days according to a previously described procedure. ${ }^{30}$ Eighty rats were randomly divided into eight groups: Group A (normal control group) received tap water for 14 days; Group B (model control group) received DSS $(5 \% \mathrm{w} / \mathrm{v})$ in drinking water for 6 days, after which the animals received regular tap water for 7 days; and Group C received DSS (5\% w/v) 
water for 6 days followed by treatment with sulfasalazine (SASP suspended in $0.5 \% \mathrm{w} / \mathrm{v}$ normal saline, $100 \mathrm{mg} / \mathrm{kg}$ ). Groups D-H received DSS (5\% w/v) similar to group C. Group D received GPs (equivalent to $50 \mathrm{mg} / \mathrm{kg}$ AT-1), group E received EPs (equivalent to $200 \mathrm{mg} / \mathrm{kg} \mathrm{BBR}$ ) for 7 days after DSS administration, and group F-H received DPs (equivalent to $50 \mathrm{mg} / \mathrm{kg}$ AT- 1 and $50,100,200 \mathrm{mg} / \mathrm{kg}$ BBR) for 7 days after DSS administration. The rats were treated once per day P.O. for 7 days (groups A and $B$ received normal saline instead of treatment drug), and their body weight was recorded daily. The beginning of the UC model induction was considered day 1. Rat body weight was recorded daily. Blood samples collected from the rats via the eye retro-orbital vein were used to determine IL-1 $\beta$, IL-4, IL-6, TNF- $\alpha$, MPO, IgA and IgG concentrations. The rats were sacrificed by cervical dislocation on day 14. The colon length was measured from the ileocaecal junction to the anal verge. After that, the colon tissues were collected for scoring, gross morphology, histopathological analysis and determination of the expression levels of various inflammatory factors. In addition, the spleen and thymus were isolated to calculate the spleen and thymus index.

\section{Colon/Body Weight Ratio}

The dissected colon tissues were opened longitudinally along the mesenteric edge and washed with ice-cold physiological saline to remove luminal content before weighing. The colon/body weight ratio ( $\mathrm{C} / \mathrm{B}$ ratio) was an index used to quantify the inflammation and was calculated as a quotient of the $10-\mathrm{cm}$ colon wet weight compared with the total body weight of each rat. ${ }^{31}$

\section{Evaluation of Clinical Colitis Activity}

The clinical activity of colitis, including weight loss, stool consistency and rectal bleeding, was evaluated to confirm the disease activity index (DAI). The DAI was determined by calculating the average of the above three parameters on a scale of $0-4$ based on the criteria shown in Table $\mathrm{S} 3 .^{32-34}$

\section{Assessment of Macroscopic Injury}

For assessment of macroscopic injury, the whole distal colon was examined by an independent observer who was unaware of the research groups. The criteria and scale employed to grade macroscopic injury are described in Table S4. ${ }^{35,36}$

\section{Histological Evaluation}

A histological scoring system was used to evaluate microscopic colonic changes. Colon tissue samples of $1 \mathrm{~cm}$ cut from the distal colon were prepared as a "Swiss roll" for fulllength histopathology evaluation. The "Swiss roll" of each group was immersed in 10\% neutral formaldehyde and subsequently embedded in paraffin. Sections of $5 \mu \mathrm{m}$ thickness were stained with haematoxylin and eosin (H\&E) to assess the degree of inflammation. The slides were then examined in a blinded fashion by a pathologist based on a scale that graded the presence of oedema, erosion-ulceration, crypt damage and inflammatory area percentage using a standard light microscope as described previously. The basis of inflammation scoring based on assessment of microscopic $(100 \times)$ histological changes is shown in Table S5..$^{37,38}$

\section{Determination of IL-I $\beta$, IL-4, IL-6, TNF- $\alpha, \operatorname{Ig} A$, and IgG Levels and MPO Activity}

Cytokines are considered crucial signals in the intestinal immune system, and immune cells, such as $\mathrm{T}$ cells, dendritic cells, macrophages, and intestinal epithelial cells, are involved in the secretion of various cytokines that regulate the inflammatory response in UC. ${ }^{39}$ Thus, the levels of IL-1 $\beta$ (Multi Sciences Rat IL-1 $\beta$ ELISA Kit, Hangzhou, China), IL-4 (Multi Sciences Rat IL-4 ELISA Kit, Hangzhou, China), IL-6 (Multi Sciences Rat IL-6 ELISA Kit, Hangzhou, China) and TNF- $\alpha$ (Thermo Fisher Rat TNF- $\alpha$ ELISA Kit, Vienna, Austria) in the serum and colon tissue samples were determined according to the manufacturer's instructions using the appropriate ELISA Kits. MPO (Cusabio Rat MPO ELISA Kit, Wuhan, China), IgA (Multi Sciences Rat IgA ELISA Kit, Hangzhou, China) and IgG (Multi Sciences Rat IgG ELISA Kit, Hangzhou, China) levels in the serum samples were also measured using commercially available ELISA kits according to the manufacturers' instructions. One unit of MPO activity was defined as the quantity of enzyme able to convert $1 \mu \mathrm{mol}$ of hydrogen peroxide to water in $1 \mathrm{~min}$ at room temperature. The results are expressed in $\mathrm{U} / \mathrm{g}$ of tissue.

\section{Statistical Analysis}

All data are expressed as the mean $\pm \mathrm{SD}$, and statistical analysis was performed with SPSS 20.0 statistical software. Comparisons between groups of parametric data were made using one-way analysis of variance (ANOVA) followed by Bonferroni's test. Nonparametric statistical analysis was performed with a Kruskal-Wallis test 
followed by a Mann-Whitney test to assess differences between groups. A threshold of $P<0.05$ was defined as statistically significant.

\section{Results}

\section{BBD-RSM-Based Enteric Coating Optimization and Pellet Preparation}

The experimental runs with independent variables and the observed responses for the 17 formulations are shown in Table 2. Based on the Box-Behnken model, the factor combinations resulted in different drug release rates for EPs. The cumulative release rate within $6 \mathrm{~h}$ was very low (below 10\%), indicating that the main limiting factor was the barrier action of the enteric coating. However, the cumulative release rate in PBS buffer ( $\mathrm{pH} \mathrm{7.6,6-12} \mathrm{h)} \mathrm{was} \mathrm{much} \mathrm{higher} \mathrm{(more} \mathrm{than}$ $70 \%$ ). To determine the levels of factors that yield optimum dissolution responses, mathematical relationships were generated between the dependent and independent variables using the experimental design software Design-Expert 8.0. The resulting equations in terms of coded factors for all the responses are given as follows:

$$
\begin{aligned}
Y_{1}= & 3.72-0.57 X_{1}-0.20 X_{2}-0.15 X_{3}+0.075 X_{1} X_{2} \\
& -0.025 X_{1} X_{3}+0.075 X_{2} X_{3}-0.22 X_{1}^{2}-0.073 X_{2}^{2} \\
& -0.22 X_{3}^{2}\left(R^{2}=0.9538\right)
\end{aligned}
$$

Table 2 Experimental Runs and Observed Response Values for the Box-Behnken Design

\begin{tabular}{|l|c|c|l|c|c|c|}
\hline \multirow{2}{*}{ Run } & $\boldsymbol{X}_{\mathbf{1}}$ & $\boldsymbol{X}_{\mathbf{2}}$ & $\boldsymbol{X}_{\mathbf{3}}$ & \multicolumn{3}{|c|}{ Cumulative Drug Release (\%) } \\
\cline { 5 - 7 } & & & & $\boldsymbol{Y}_{\mathbf{1}}$ & $\boldsymbol{Y}_{\mathbf{2}}$ & $\boldsymbol{Y}_{\mathbf{3}}$ \\
\hline 1 & 2 & 10 & 20 & 2.5 & 6.5 & 70.38 \\
2 & 1.25 & 15 & 20 & 3.2 & 8.1 & 73.27 \\
3 & 1.25 & 10 & 15 & 3.9 & 13.9 & 77.69 \\
4 & 2 & 5 & 15 & 2.9 & 7.8 & 72.50 \\
5 & 1.25 & 10 & 15 & 3.6 & 12.7 & 76.84 \\
6 & 0.5 & 10 & 10 & 4 & 17.1 & 82.28 \\
7 & 2 & 15 & 15 & 2.7 & 9.5 & 73.78 \\
8 & 1.25 & 15 & 10 & 3.2 & 14.6 & 75.05 \\
9 & 2 & 10 & 10 & 3 & 11.7 & 72.33 \\
10 & 1.25 & 5 & 10 & 3.8 & 16.8 & 80.66 \\
11 & 1.25 & 10 & 15 & 3.9 & 14.3 & 76.84 \\
12 & 1.25 & 10 & 15 & 3.6 & 12.9 & 75.56 \\
13 & 0.5 & 10 & 20 & 3.6 & 10.2 & 78.54 \\
14 & 1.25 & 5 & 20 & 3.5 & 9.7 & 77.01 \\
15 & 0.5 & 15 & 15 & 3.8 & 11.8 & 79.22 \\
16 & 1.25 & 10 & 15 & 3.6 & 13.5 & 76.24 \\
17 & 0.5 & 5 & 15 & 4.3 & 16.2 & 81.68 \\
\hline
\end{tabular}

$$
\begin{aligned}
Y_{2}= & 13.46-2.48 X_{1}-0.81 X_{2}-3.21 X_{3}+1.52 X_{1} X_{2} \\
& +0.43 X_{1} X_{3}+0.15 X_{2} X_{3}-1.53 X_{1}^{2}-0.61 X_{2}^{2} \\
& -0.55 X_{3}^{2}\left(R^{2}=0.9844\right)
\end{aligned}
$$

$$
\begin{aligned}
Y_{3}= & 76.64-4.09 X_{1}-1.32 X_{2}-1.39 X_{3}+0.93 X_{1} X_{2} \\
& +0.45 X_{1} X_{3}+0.47 X_{2} X_{3}-0.23 X_{1}^{2}+0.39 X_{2}^{2} \\
& -0.52 X_{3}^{2}\left(R^{2}=0.9324\right)
\end{aligned}
$$

Eqs. (2)-(4) represent the quantitative effect of the formulation independent variables $X_{1}-X_{3}$ on the three dependent responses $Y_{1}-Y_{3}$, respectively. The independent values of $X_{1}-X_{3}$ were ascertained in Eqs. (2)-(4) to obtain the predicted values of $Y_{1}-Y_{3}$. Furthermore, the relationship between the independent variables and dependent variables was illuminated and analysed using the threedimensional (3D) response surface and two-dimensional (2D) contour plots shown in Figure 1.

As seen from Figure 1, when $X_{1}, X_{2}$ and $X_{3}$ increased, $Y_{1}$, $Y_{2}$ and $Y_{3}$ increased as well. It was found that the ratio of EUDRAGIT ${ }^{\circledR} \mathrm{FS} 30 \mathrm{D}$ to EUDRAGIT ${ }^{\mathbb{B}} \mathrm{L}$ 30D-55 $\left(X_{1}\right)$, plasticizer concentration $\left(X_{2}\right)$ and coating weight gain $\left(X_{3}\right)$ were significant factors affecting $Y_{1}, Y_{2}$ and $Y_{3}$ (the cumulative drug release in $2 \mathrm{~h}, 6 \mathrm{~h}$ and $12 \mathrm{~h}$ ). The model predicted $Y_{1}, Y_{2}$ and $Y_{3}$ values of 2.50, 6.34 and 70.92 when $X_{1}, X_{2}$ and $X_{3}$ were 2.0, 11.7 and 20.0, respectively. The results of three validation experiments showed that the predicted values and observed values were in reasonably close agreement (Table 3 ). ${ }^{40}$

\section{Characterization of the $\beta-C D$ Inclusion Complex}

Figure $2 \mathrm{~A}$ shows DSC curves of $\beta-C D$, volatile oil $/ \beta-C D$ physical mixture, volatile oil and the volatile oil $/ \beta-C D$ inclusion complex. The thermogram of $\beta$-CD showed a wide endothermic peak at approximately $145.48{ }^{\circ} \mathrm{C}$, which referred to water release. There was another endothermic peak at approximately $314.24{ }^{\circ} \mathrm{C}$, which was mainly related to the phase transition of $\beta-\mathrm{CD}$. Volatile oil exhibited an endothermal effect peaking at approximately $307.40^{\circ} \mathrm{C}$, which was attributed to the decomposition of the volatile oil. The thermogram of the physical mixture was similar to the superimposition of the individual thermograms of $\beta-C D$ and volatile oil. However, the thermogram of the inclusion complex exhibited a different pattern, and the two characteristic peaks of $\beta-\mathrm{CD}$ disappeared, indicating that water molecules in the cavity of $\beta-C D$ were displaced. ${ }^{41}$ Furthermore, a new characteristic peak 
A
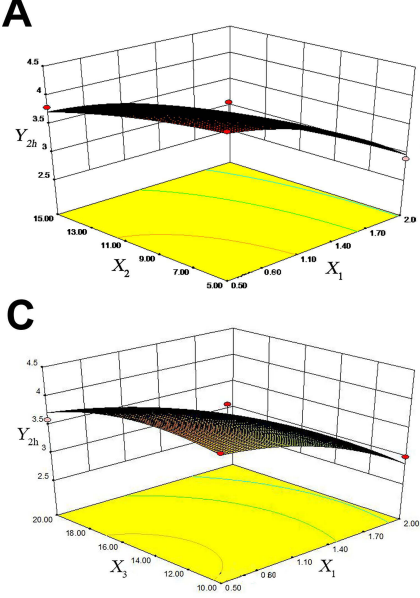

E

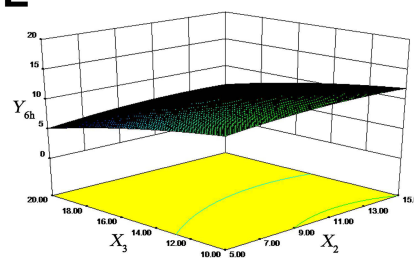

G

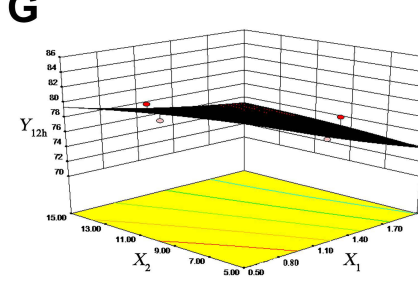

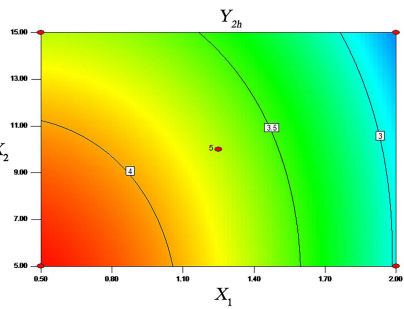

$Y_{2 \mathrm{~h}}$

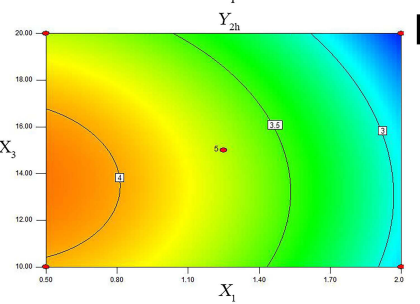

$Y_{6 \mathrm{~h}}$

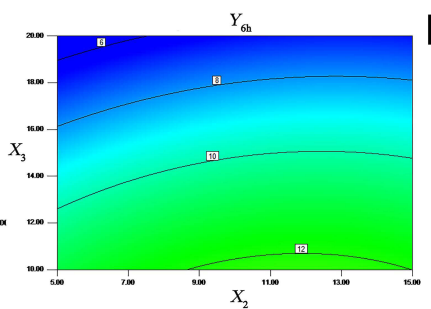

$Y_{12 \mathrm{~h}}$

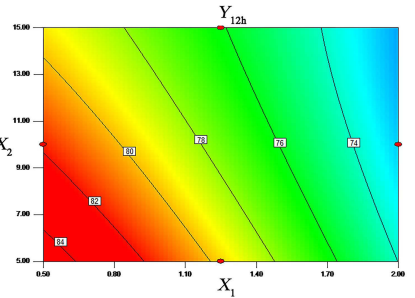

I

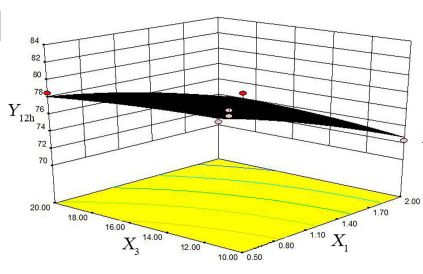

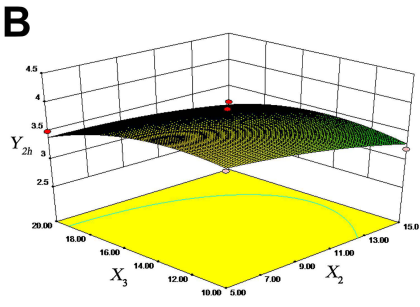

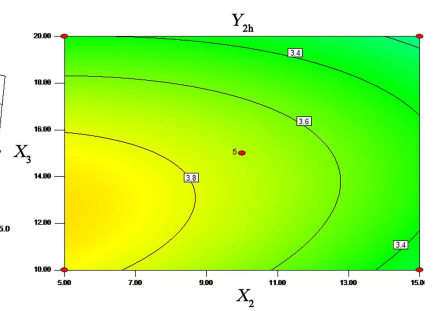

D

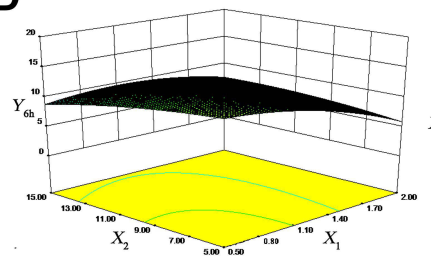

F

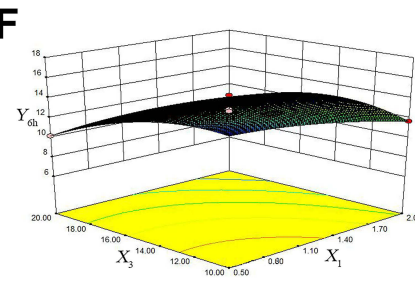

H

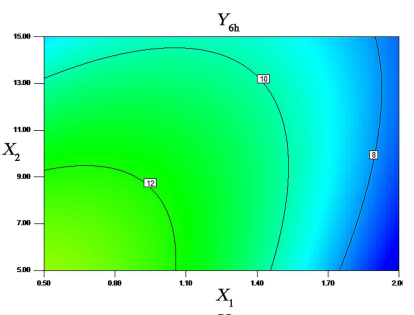

$Y_{6}$

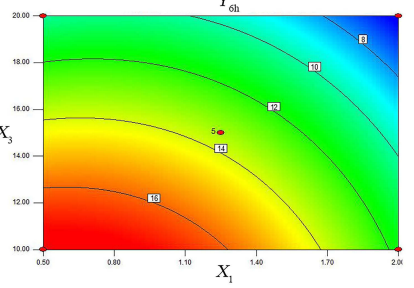

$Y_{12 \mathrm{~b}}$

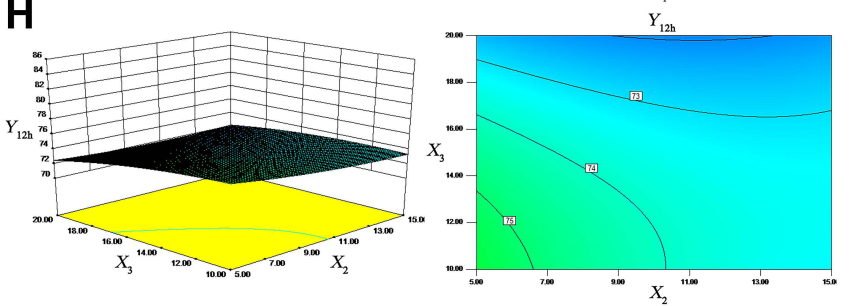

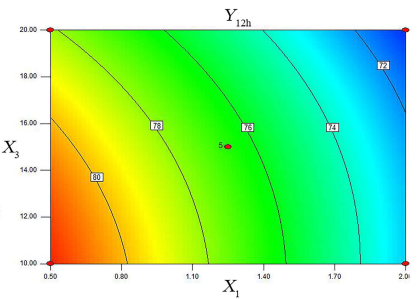

Figure I Effect of variables $\left(X_{1}-X_{3}\right)$ on the response $\left(Y_{1}-Y_{3}\right)$ : 3D response surface plots and $2 \mathrm{D}$ contour plots. $X_{1}$ : ratio of EUDRAGIT ${ }^{\circledR} \mathrm{FS} 30 \mathrm{D}$ to EUDRAGIT ${ }^{\circledR} \mathrm{L} 30 \mathrm{D}-55$; $X_{2}$ : plasticizer concentration (based on dry polymer weight); $X_{3}$ : coating weight gain. $Y_{1}-Y_{3}$ : cumulative drug release at $2 \mathrm{~h}, 6 \mathrm{~h}$ and $12 \mathrm{~h}$. (A) the effects of $X_{1}$ and $X_{2}$ on $Y_{1} ;(\mathbf{B})$ the effects of $X_{2}$ and $X_{3}$ on $Y_{1} ;(\mathbf{C})$ the effects of $X_{1}$ and $X_{3}$ on $Y_{1} ;(\mathbf{D})$ the effects of $X_{1}$ and $X_{2}$ on $Y_{2} ;(\mathbf{E})$ the effects of $X_{2}$ and $X_{3}$ on $Y_{2} ;(\mathbf{F})$ the effects of $X_{1}$ and $X_{3}$ on $Y_{2} ;(\mathbf{G})$ the effects of $X_{1}$ and $X_{2}$ on $Y_{3} ;(\mathbf{H})$ the effects of $X_{2}$ and $X_{3}$ on $Y_{3} ;(\mathbf{I})$ the effects of $X_{1}$ and $X_{3}$ on $Y_{3}$.

appeared at $277.49^{\circ} \mathrm{C}$, which we speculated was due to the occurrence of an intermolecular interaction that generates a new phase rather than the volatile oil and $\beta-C D$. All these

Table 3 Comparison Between the Observed and Predicted Responses of the Optimized Enteric Coating Layer

\begin{tabular}{|l|c|c|c|}
\hline $\begin{array}{l}\text { Evaluating } \\
\text { Indexes }\end{array}$ & Observed & Predicted & $\begin{array}{c}\text { Prediction Error } \\
\text { (\%) }\end{array}$ \\
\hline$Y_{1}$ & 2.63 & 2.50 & $5.47 \%$ \\
$Y_{2}$ & 6.55 & 6.34 & $3.26 \%$ \\
$Y_{3}$ & 73.24 & 70.92 & $3.27 \%$ \\
\hline
\end{tabular}

results indicate that the volatile oil was wrapped in the cavity of $\beta-\mathrm{CD}$.

$\mathrm{X}$-ray powder diffraction can be used to determine the crystal type of crystal compounds. As shown in Figure 2B, $\beta-\mathrm{CD}$ exhibited many crystalline peaks between $5^{\circ}$ and $50^{\circ}(2 \theta=8.88,12.47,18.76$ and $27.05^{\circ}$ ), indicating that $\beta-\mathrm{CD}$ mainly existed in a crystalline form. Volatile oil showed a large broad peak, suggesting that it is in an amorphous state. The XRD pattern of the physical mixture showed approximate superimposition of the individual patterns of $\beta-\mathrm{CD}$ and volatile oil. The inclusion complex exhibited different 
A

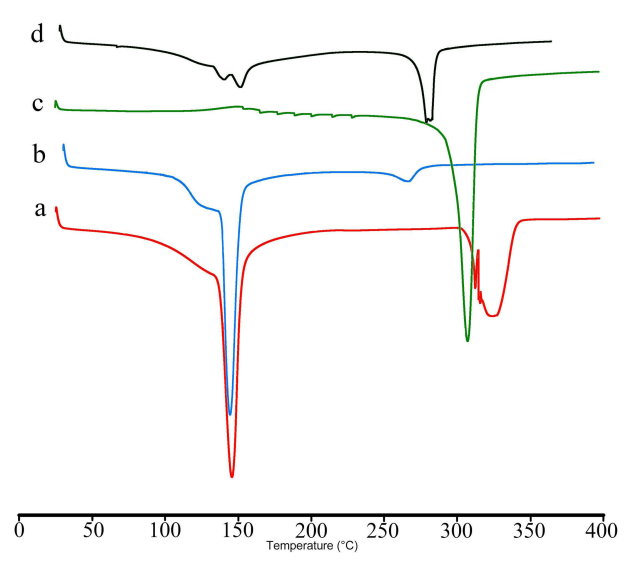

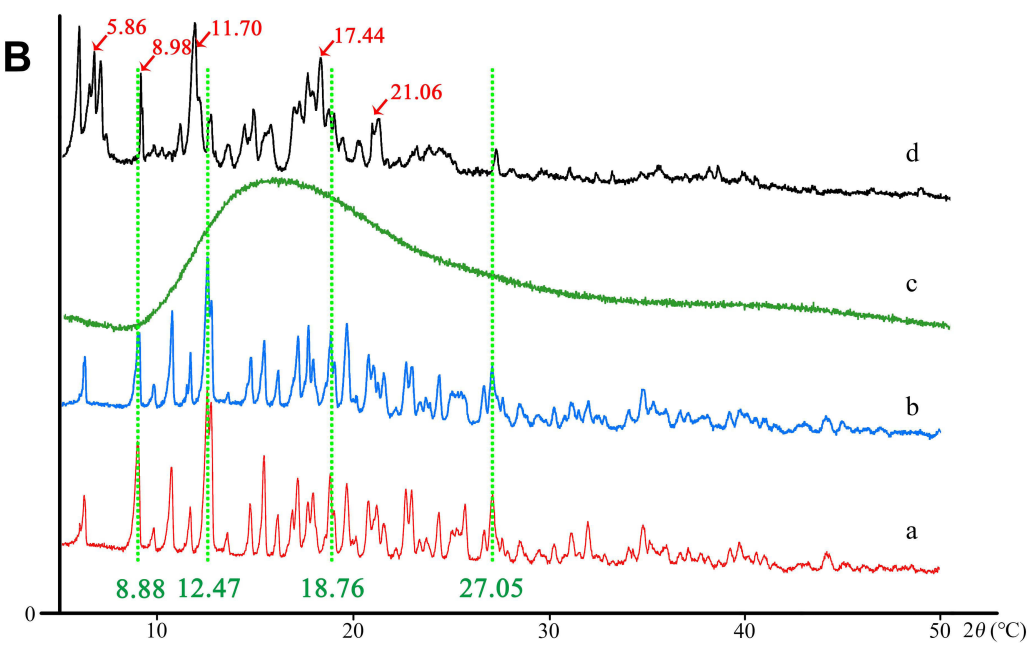

Figure 2 Characterization of the $\beta-C D$ inclusion complex using DSC (A) and XRD (B): (a) $\beta-C D$; (b) volatile oil/ $\beta-C D$ physical mixture; (c) volatile oil; (d) volatile oil/ $\beta-C D$ inclusion complex.

main peaks $\left(2 \theta=5.86,8.98,11.70,17.44\right.$ and $\left.21.06^{\circ}\right)$ and distinctive peak patterns, with relatively broad bands compared with the physical mixture. ${ }^{41}$ The appearance of these new characteristic peaks indicated that the inclusion complex was a new crystal form.

\section{Characterization of Pellets}

The WG $(\%)$, yield $(\%), \mathrm{RSD}_{\mathrm{w}}(\%)$ and coating loss $(\%)$ for GPs, sub-Ps and EPs are listed in Table S2. The results showed that the preparation process employed for GPs, Sub-Ps and EPs was reasonable and repeatable and that the process stability was admirable.
According to the naked eye and SEM observations, the GPs were white and spherical or ellipsoid, while the EPs were yellow and brown spheres with smooth and round surfaces (Figure $3 \mathrm{~A}$ and $\mathrm{C}$ ( $\mathrm{a}$ and $\mathrm{b}$ )). Investigation of the morphology of these two pellets indicated that both GPs and EPs have a uniform size, ideal narrow size distribution, regular round shape and smooth surface.

The obtained particle size distribution of the coated pellets is presented in Table 4. The majority of the GPs and EPs lay within the intervals 700-830 $\mu \mathrm{m}$ (24-20 mesh) and $880-1180 \mu \mathrm{m}$ (18-14 mesh). SEM images of
A

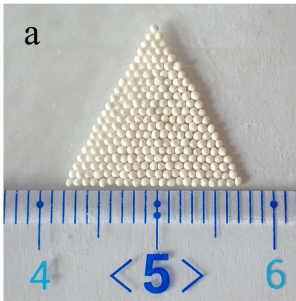

b

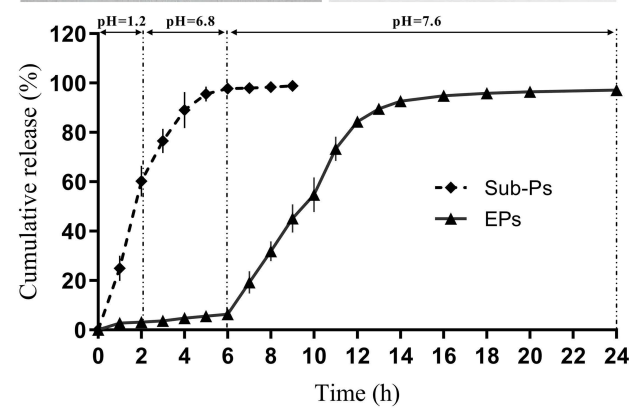

C
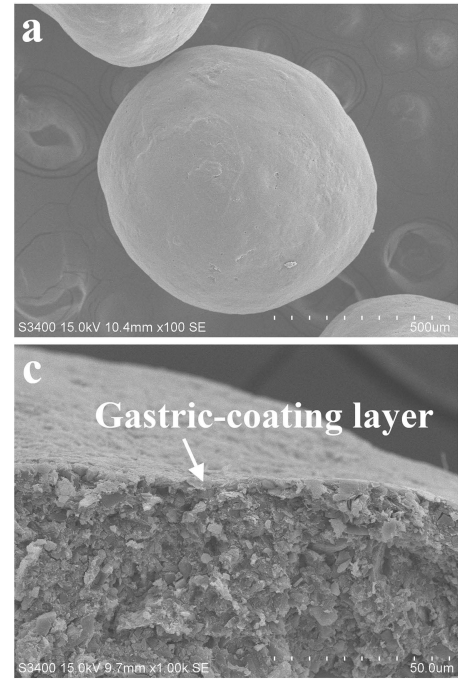
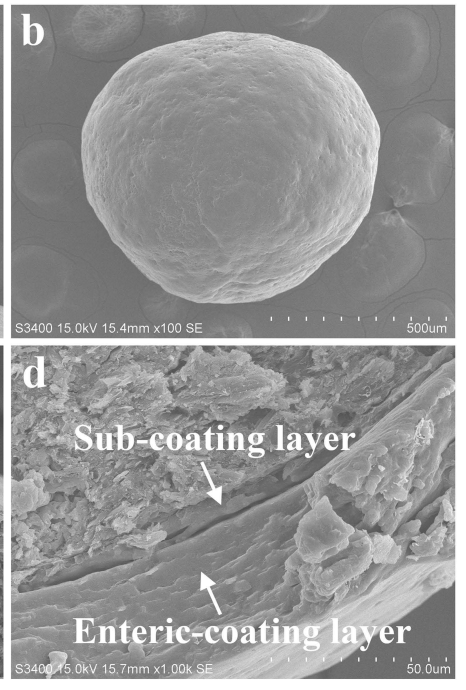

Figure 3 Physicochemical characterization of pellets. (A) Pellet size and shape observed by the naked eye. (a) GPs and (b) EPs (B) in vitro release profiles of Sub-Ps and EPs in different media ( $\mathrm{pH}=1.2$ for $2 \mathrm{~h}, \mathrm{pH}=6.8$ for $4 \mathrm{~h}, \mathrm{pH}=7.6$ for $18 \mathrm{~h}$ ) (C) magnified scanning electron microscopy photograph of pellets. (a) Complete GPs and (b) complete EPs at 100x; (c) GPs cross-sections and (d) EPs cross-sections at 1000x in dissolution medium. 
Table 4 The Particle Size Distribution of GPs and EPs $(n=3)$

\begin{tabular}{|l|c|c|c|}
\hline \multicolumn{2}{|l|}{ GPs } & \multicolumn{2}{|c|}{ EPs } \\
\hline $\begin{array}{l}\text { Size } \\
\text { (Mestribution }\end{array}$ & $\begin{array}{c}\text { Ratio (\%) } \\
\text { Ristribution } \\
\text { (Mesh) }\end{array}$ & $\begin{array}{c}\text { Mass } \\
\text { Ratio (\%) }\end{array}$ \\
\hline$<30$ & 5 & $<20$ & 7 \\
$30-24$ & 18 & $18-20$ & 11 \\
$24-20$ & 65 & $14-18$ & 61 \\
$20-18$ & 8 & $12-14$ & 16 \\
$>18$ & 4 & $>12$ & 5 \\
\hline
\end{tabular}

pellet cross-sections showing the structure of the inner region of the pellets are presented in Figure 3C (c). The sub coating layer (Figure 3C (d)) and polymer layer can be clearly observed in the cross-section views, indicating that the pellet core had a compact structure with the polymer layer tightly coated on the core.

Roundness, friability and bulk density are three important product quality requirements, and these parameters are presented in Table 5. The lower plane-critical angle and friability were related to the superior roundness and indicated that both GPs and EPs had good rigidity to avoid being smashed during the fluid-bed coating procedure.

HPLC-QQQ-MS and HPLC content determination methods were established for BBR and AT-1 to calculate drug loading and in vitro drug release. The good linearity of the determination method and the RSD of intraday/ interday precision had a value smaller than $2 \%$ for each concentration level, which demonstrates good precision of the measurement. On this premise, the BBR and AT-1 loading in EPs and GPs was $125.61 \pm 0.1 \mathrm{mg} / \mathrm{g}$ and $17.11 \pm 0.16 \mathrm{mg} / \mathrm{g}$, respectively.

The release profiles of the Sub-Ps and EPs are shown in Figure 3B. BBR release from the Sub-Ps without an enteric layer was immediate and almost complete within $4 \mathrm{~h}$, demonstrating that Sub-Ps are not appropriate for colon drug delivery. Sufficient acid resistance was achieved when the Sub-Ps were coated with an enteric coating layer optimized by BBDRSM. The cumulative release of BBR in artificial gastric

Table 5 Physical Characteristics of GPs and EPs $(n=3$, Mean \pm SD)

\begin{tabular}{|l|c|c|}
\hline Parameters & GPs & EPs \\
\hline Roundness $\left(\theta /{ }^{\circ}\right)$ & $12.17 \pm 0.35$ & $9.73 \pm 1.50$ \\
Friability $(\mathrm{Fr} / \%)$ & $3.87 \pm 0.65$ & $4.67 \pm 0.45$ \\
Bulk density $\left(\mathrm{g} / \mathrm{cm}^{3}\right)$ & $0.62 \pm 0.030$ & $0.76 \pm 0.06$ \\
\hline
\end{tabular}

medium ( $\mathrm{pH} 1.2$ ) for $2 \mathrm{~h}$ was less than $5 \%$ and that in artificial intestinal medium ( $\mathrm{pH} 6.8$ ) for $4 \mathrm{~h}$ was less than $10 \%$. Exhilaratingly, almost $95 \%$ of BBR release occurred within $10 \mathrm{~h}$ thereafter in an artificial colonic medium $(\mathrm{pH}$ 7.6). The increase in the $\mathrm{pH}$ of the dissolution fluid ( $\mathrm{pH} 7.6)$ significantly accelerates the rate of dissolution.

\section{In vivo Imaging-Based Colon-Targeted Study of EPs}

The results of the in vivo targeting evaluation of EPs are shown in Figure 4. Compared with coated pellets, the uncoated fluorescent pellets showed large dot-like fluorescence 1-2 $\mathrm{h}$ after oral administration, indicating that they began to disintegrate after reaching the stomach. At 4-6 $\mathrm{h}$ after oral administration, the entire small intestine treated with uncoated pellets was filled with fluorescence, indicating that most of the pellets had been disintegrated by this time. Eight to ten hours later, the fluorescence in the colon from the uncoated pellets gradually weakened due to their complete disintegration, and the fluorescein was quenched by the contents of the intestinal tract. However, the segment of the intestine from the ileocaecal region to the end of the colon was filled with fluorescence after oral administration of enteric coated fluorescent pellets, which means that a large number of pellets disintegrated and released the drug.

\section{In vivo Pharmacokinetics Study}

A pharmacokinetic study in SD rats after oral administration of DPs and BPC was performed, and the mean plasma concentration-time curve was constructed as shown in Figure 5. Lists of the corresponding pharmacokinetic parameters are shown in Table 6. As shown in Figure 5A, AT-1 can be quickly absorbed into the blood after oral administration of BPC and DPs. The $T_{\max }$ values of the two formulations were the same at $1.00 \pm 0.00 \mathrm{~h}$. The $C_{\max }$ values of BPC and DPs were $109.49 \pm 5.61 \mathrm{ng} / \mathrm{mL}$ and $106.94 \pm 11.43 \mathrm{ng} / \mathrm{mL}$, respectively $(P>0.05)$. These results indicate that inclusion of $\beta$-CD increases the solubility and dissolution of active ingredients in volatile oils. However, the $T_{\max }$ of AT-1 showed a "bimodal" phenomenon, indicating that there may be secondary absorption in the blood. Interestingly, the $\mathrm{AUC}_{0 \rightarrow \mathrm{t}}$ value in the DPs group increased slightly compared with that in the BPC group, and the relative bioavailability value was $153.13 \%$. Furthermore, the pattern showed a consistent plasma concentration of AT-1, indicating higher bioavailability in the DPs group. In addition, the DPs group had a higher MRT 


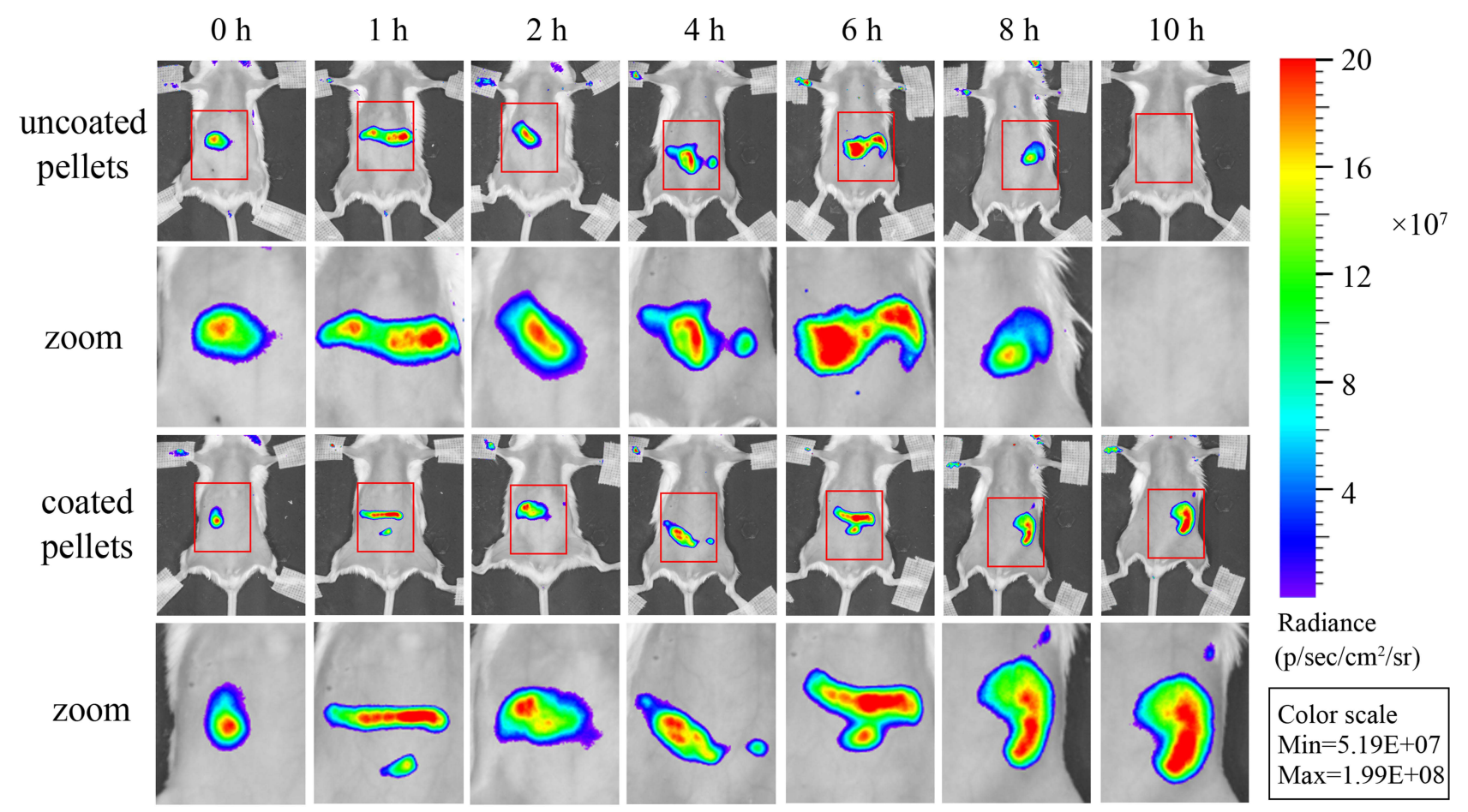

Figure 4 In vivo imaging of fluorescent colon-targeted pellets in mice.

A

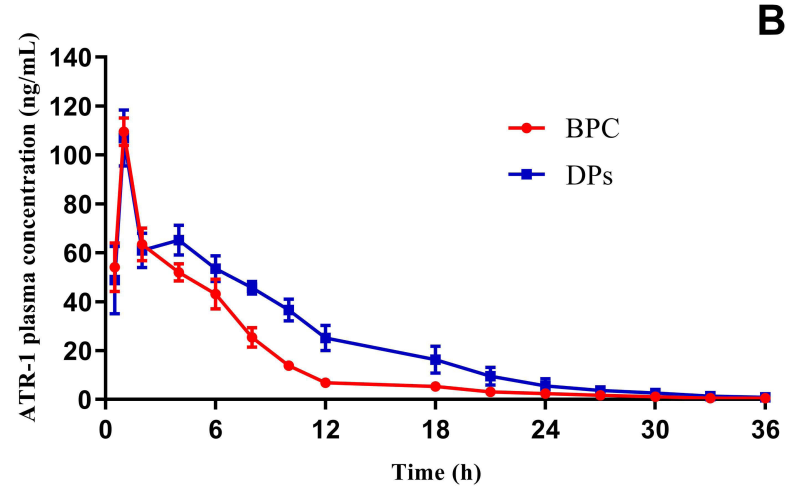

B

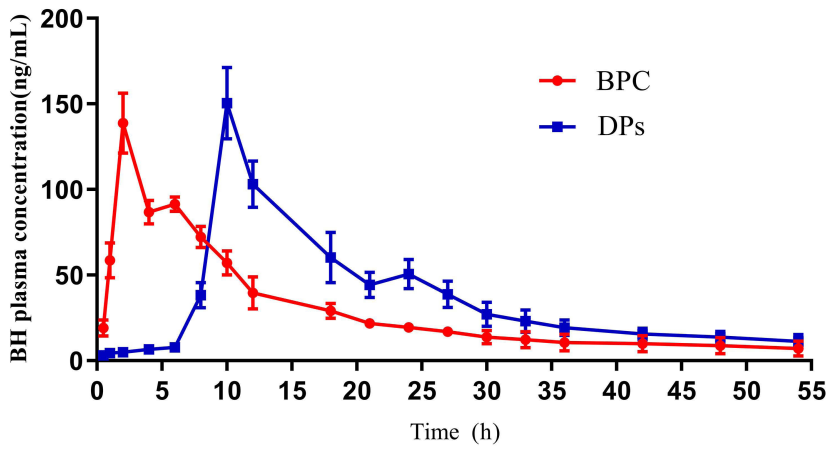

Figure 5 Pharmacokinetic profile of AT-I (A) and BBR (B) in SD rats after oral administration of BPC and DPs at a single dose of 50 mg of AT-I and 200 mg of BBR. Each value represents the mean $\pm S D(n=3)$.

value than the BPC group, which means that AT-1 release from the pellets exhibited a certain sustained release effect.

According to Figure 5B, the $T_{\max }$ values of BBR in plasma from the BPC group and DPs group were $2.00 \pm 0.00$ and $10.00 \pm 0.00 \mathrm{~h}$, respectively. Furthermore, the obvious time lag in the DPs group indicated that the coated pellets had a good controlled release and a colon-specific effect. The $T_{\max }$ of both groups showed a "double peak" phenomenon due to enterohepatic circulation during BBR absorption. ${ }^{42}$ The $C_{\max }$ of the DPs group was significantly different from that of the BPC group. The relative bioavailability in the two experimental groups was $121.38 \%$, indicating a higher relative bioavailability of the DPs. In addition, the plasma drug concentration-time curve was gentler, and the DPs group had a higher MRT value than the BPC group, indicating an obvious sustained release effect in the DPs group, which can better stabilize the blood drug concentration and thus increase bioavailability.

\section{Anti-Ulcerative Colitis Analysis Effect of UC on Body Weight}

Figure 6 shows that the body weight of rats in healthy control group A slightly increased over time. All the DSS- 
Table 6 Pharmacokinetic Parameters of AT-I and BBR Delivered by DPs and BPC After Oral Administration ( $n=3$, Mean \pm SD)

\begin{tabular}{|l|c|c|c|c|}
\hline \multirow{2}{*}{ Parameters } & \multicolumn{2}{|c|}{ AT-I } & \multicolumn{2}{c|}{ BBR } \\
\cline { 2 - 5 } & BPC & DPs & BPC & $150.43 \pm 20.82$ \\
\hline$C_{\max }(\mathrm{ng} / \mathrm{mL})$ & $109.49 \pm 5.61$ & $106.94 \pm 11.43$ & $138.81 \pm 17.63$ & $10.00 \pm 0.00$ \\
$T_{\max }(\mathrm{h})$ & $1.00 \pm 0.00$ & $1.00 \pm 0.00$ & $2.00 \pm 0.00$ & $1928.56 \pm 297.47$ \\
$A \cup C_{0 \rightarrow \mathrm{t}}(\mathrm{ng} \mathrm{h} / \mathrm{mL})$ & $543.91 \pm 50.24$ & $832.87 \pm 130.73$ & $1588.79 \pm 160.65$ & $2393.53 \pm 418.88$ \\
$A \cup C_{0 \rightarrow \infty}(\mathrm{ng} h / \mathrm{mL})$ & $547.34 \pm 51.74$ & $839.04 \pm 134.06$ & $1888.77 \pm 524.25$ & $26.74 \pm 13.97$ \\
$T_{1 / 2}(\mathrm{~h})$ & $4.94 \pm 1.06$ & $4.1 \pm 1.54$ & $22.82 \pm 16.57$ & $36.05 \pm 12.29$ \\
MRT $(\mathrm{h})$ & $6.38 \pm 0.66$ & $8.75 \pm 0.91$ & $26.36 \pm 16.91$ & 121.38 \\
$F_{\text {rel }}(\%)$ & - & 153.13 & - & \\
\hline
\end{tabular}

Abbreviations: UC, ulcerative colitis; BBR, Berberine; CC, Coptis chinensis Franch; AM, Atractylodes macrocephala Koidz; DPs, dual-targeted pellets; OCDDS, oral colontargeted drug delivery system; DSS, dextran sulfate sodium salt; AT-I, Atractylenolide I; $\beta$-CD, $\beta$-cyclodextrin; MCC, Microcrystalline cellulose; HPMC, Hypromellose; SASP, Sulfasalazine; BBD-RSM, Box Behnken design of a response surface methodology; GPs, Gastric coated pellets; Eps, Enteric coated pellets; Sub-Ps, EPs core coated with 4\% HPMC as sub-coating layer; BPC, bulk pharmaceutical chemicals.

induced groups showed the lowest body weight on day 7 or day 8 , and the haematochezia and diarrhoea symptoms were most severe on these two days in the experimental process, which indicated that the establishment of the UC model was successful. After day 8, the body weight of rats in almost all the DSS-induced groups showed an upward trend, indicating that each drug administration group had a certain effect on alleviating the weight loss caused by UC. Among them, the DPs medium-dose group $G$ and SASP group $\mathrm{C}$ showed the most effectiveness.

\section{Effect of DPs Administration on the Clinical Activity Score in DSS-Induced UC}

As shown in Figure 7, for all the DSS-induced groups, the DAI score increased rapidly and consistently over the 6 days of the induction experiment. Starting on day
7, all drug-receiving groups showed a decrease in inflammation severity after a lag time of 24 to 48 $\mathrm{h}$ and a reduction in the DAI score. The maximum reduction was observed in the DPs medium- and highdose groups on day 14. This reflects the efficacy of the prepared DPs, and a dose of $50 \mathrm{mg} / \mathrm{kg}$ AT- $1+100 \mathrm{mg} /$ $\mathrm{kg}$ BBR in rats was inferred to be sufficient to achieve satisfactory therapeutic efficacy. In the case of group F, the DAI index was observed to be significantly lower than that of model group B on day 14 (Figure 8A), indicating a partial reversal of inflammation even at a dose of $50 \mathrm{mg} / \mathrm{kg}$ AT- $1+50 \mathrm{mg} / \mathrm{kg}$ BBR. In addition, the DAI score of groups $\mathrm{G}$ and $\mathrm{H}$ was also found to be significantly lower $(P<0.05)$ than that of SASP group $\mathrm{C}$, indicating better efficacy of DPs against inflammation induced by UC. Nevertheless, in both groups A and B,

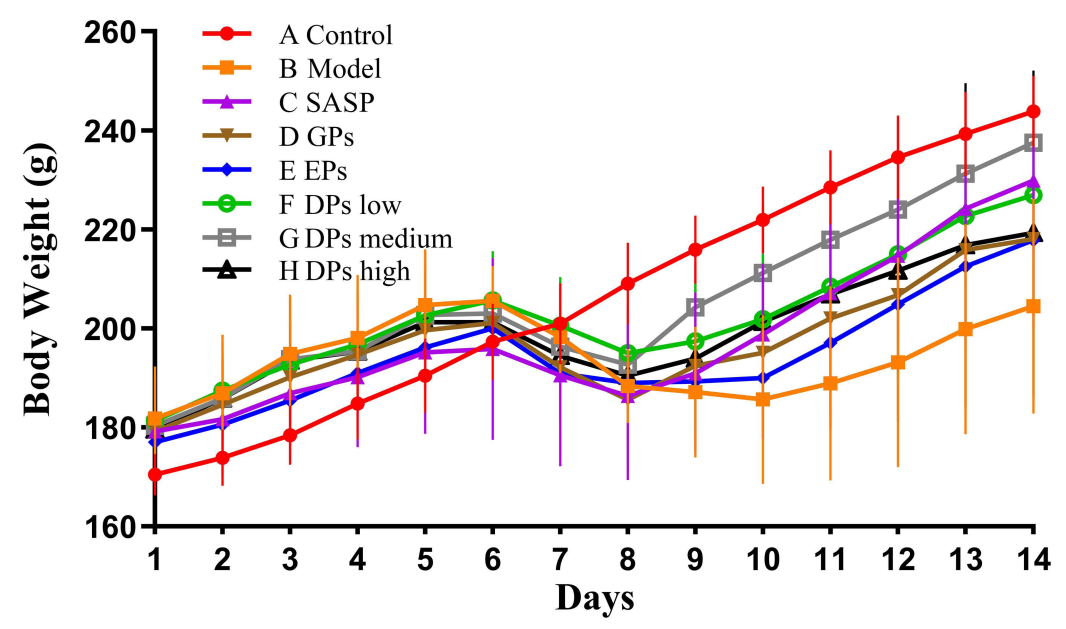

Figure 6 Time-dependent variations in body weight. (A) Control group, (B) model group, (C) SASP group, (D) GPs group, (E) EPs group, (F) DPs low-dose group, (G) DPs medium-dose group, $(\mathbf{H})$ DPs high-dose group $(\mathrm{n}=10)$. 


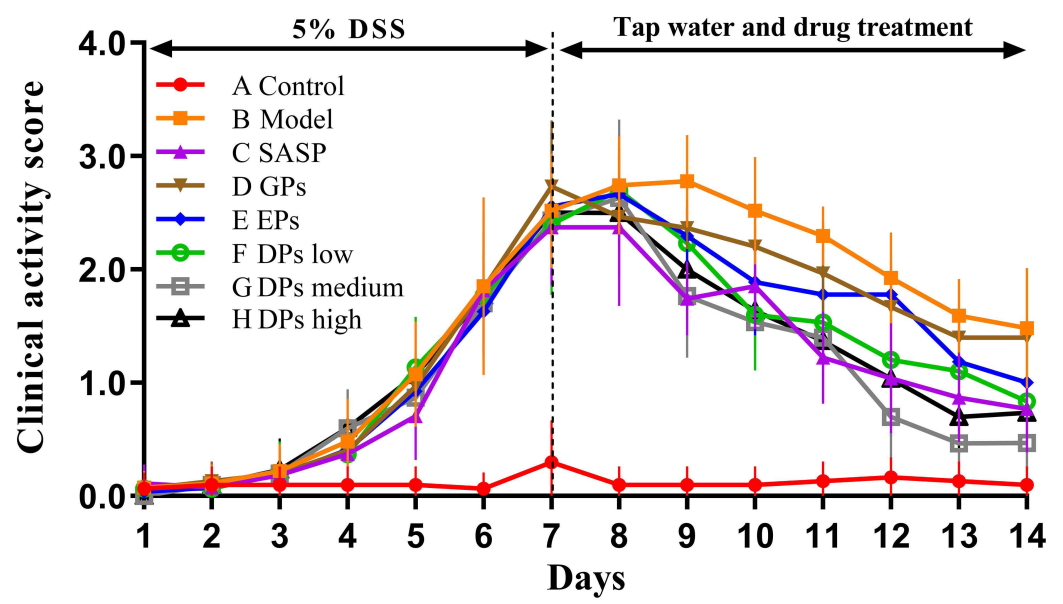

Figure 7 Clinical activity scores (DAI) throughout the entire experimental period. (A) Control group, (B) model group, (C) SASP group, (D) GPs group, (E) EPs group, (F) DPs low-dose group, $(\mathbf{G})$ DPs medium-dose group, $(\mathbf{H})$ DPs high-dose group $(n=10)$.
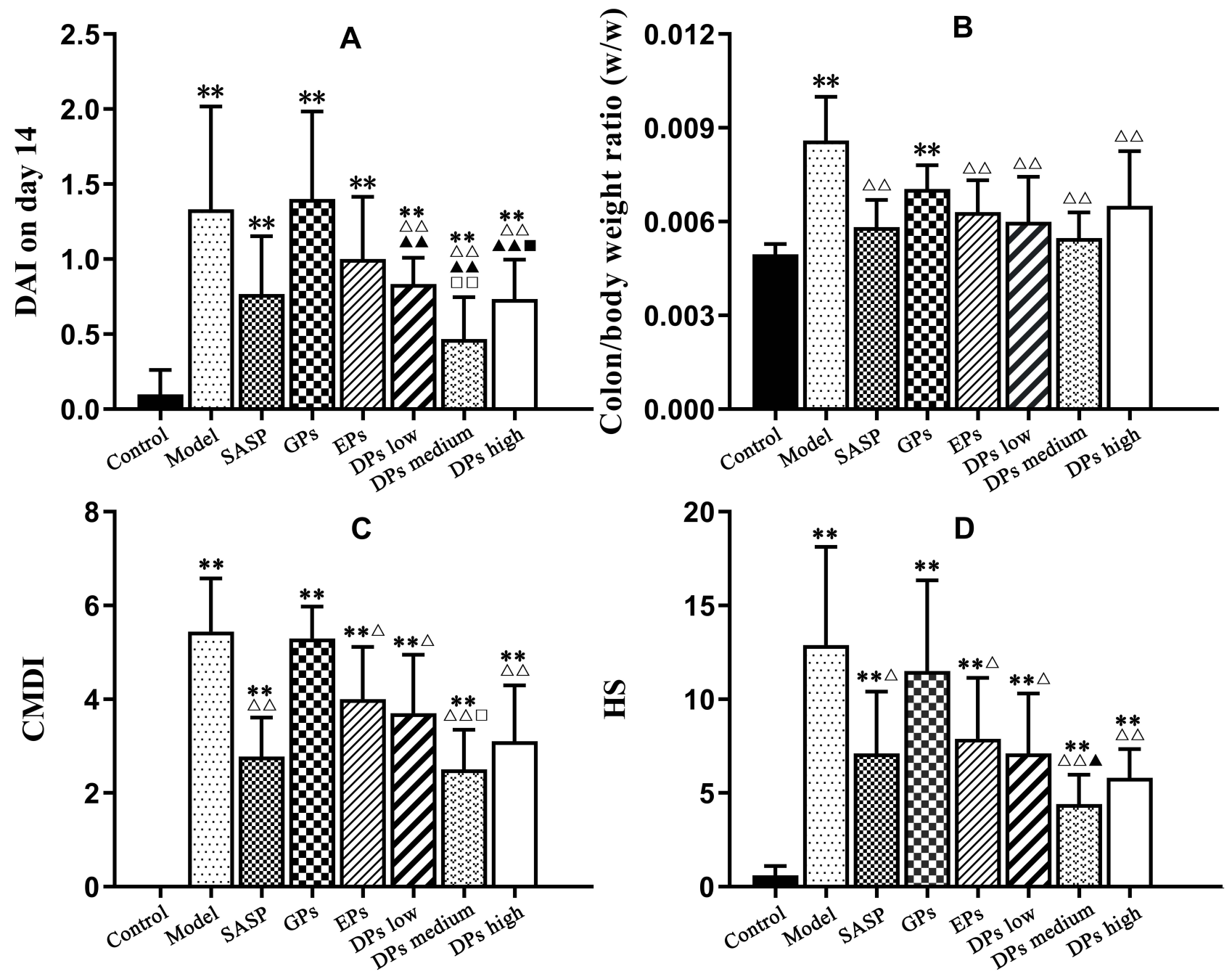

Figure 8 (A) Scoring of disease activity index (DAI) in each group, (B) The colon/body weight ratio, (C) scoring of colon mucosal damage index (CMDI) and (D) microscopic histological changes on day $14(\bar{x} \pm s, \mathrm{n}=10)$. Comparisons: ${ }^{* * P} P<0.0$ I vs control group, ${ }^{\Delta} P<0.05,{ }^{\Delta \Delta} P<0.01$ vs model group, ${ }^{\Delta} P<0.05,{ }^{\Delta}{ }^{\Delta} P<0.0$ I vs $S A S P$ group, ${ }^{\square} P<0.05,{ }^{\square} P<0.01$ vs DPs low-dose group, $" P<0.05$ vs $D P s$ medium-dose group, $* * P<0.01$ vs control group. 
the treatment was less effective in reducing the DAI score. This gives a clear indication that the DPs exhibited enhanced efficacy compared with GPs or EPs monotherapy, which resulted in a markedly reduced DAI score.

\section{Effect of DPs Administration on the Colon/Body Weight Ratio and Macroscopic Injury Score}

As shown in Figure 8B, GPs administration did not exert a significant effect; however, in the other drug administration groups, the $\mathrm{C} / \mathrm{B}$ ratio was significantly reduced.

The colon mucosal damage index (CMDI) is shown in Figure 8C. The results showed that the colorectal morphology in the rats in the control group was normal, and the inner wall of the intestine was smooth and elastic. The colour of the intestinal wall in all the DSS-induced groups was dark red. Diffuse oedema and destruction of the mucous membranes appeared in all parts of the intestinal wall. The CMDI scores of the rats in each administration group were significantly different from those in the control group $(P<0.01)$. Except for the EPs group, there were significant differences between each administration group and the model group $(P<0.05)$, indicating that each treatment administered had a certain effect in improving colorectal mucosal damage. The DPs medium-dose group had a lower score than the other administration groups, indicating that a better therapeutic effect was observed in the DPs medium-dose group.
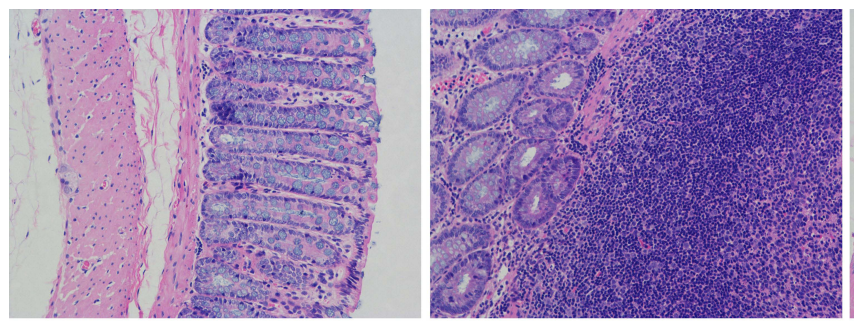

A

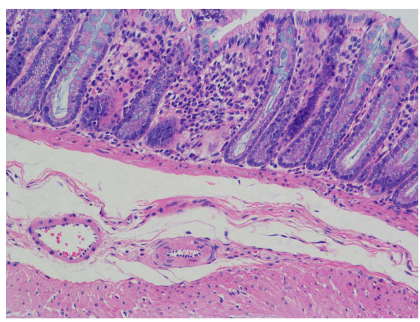

$\mathbf{E}$

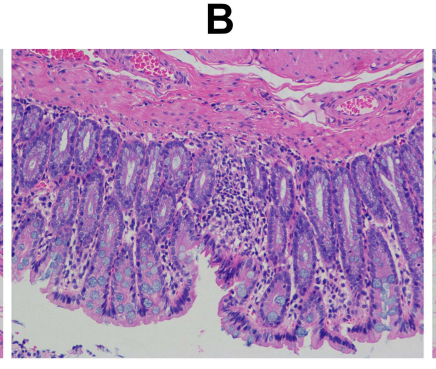

$\mathbf{F}$

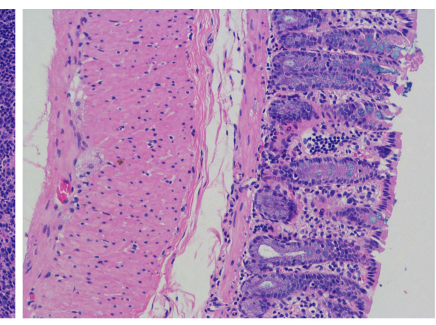

C

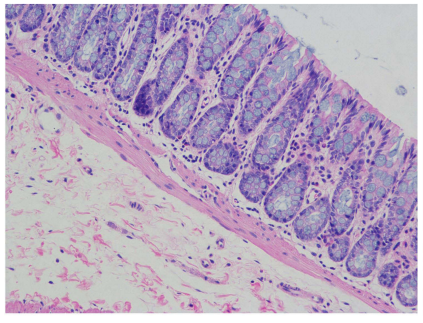

G

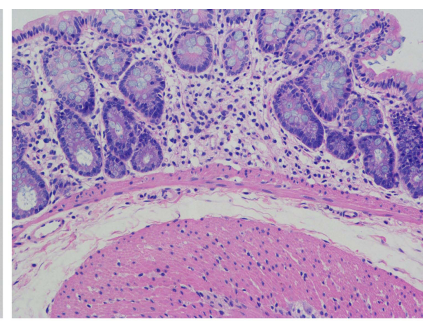

D

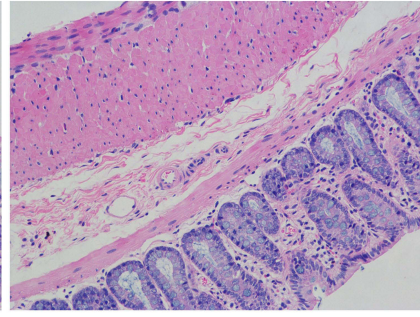

H

Figure 9 Pathological colon sections stained with H\&E in each group $(200 \times)$ (A) control group, (B) model group, (C) SASP group, (D) GPs group, (E) EPs group, (F) DPs low dose-group, (G) DPs medium-dose group, (H) DPs high-dose group. 
A
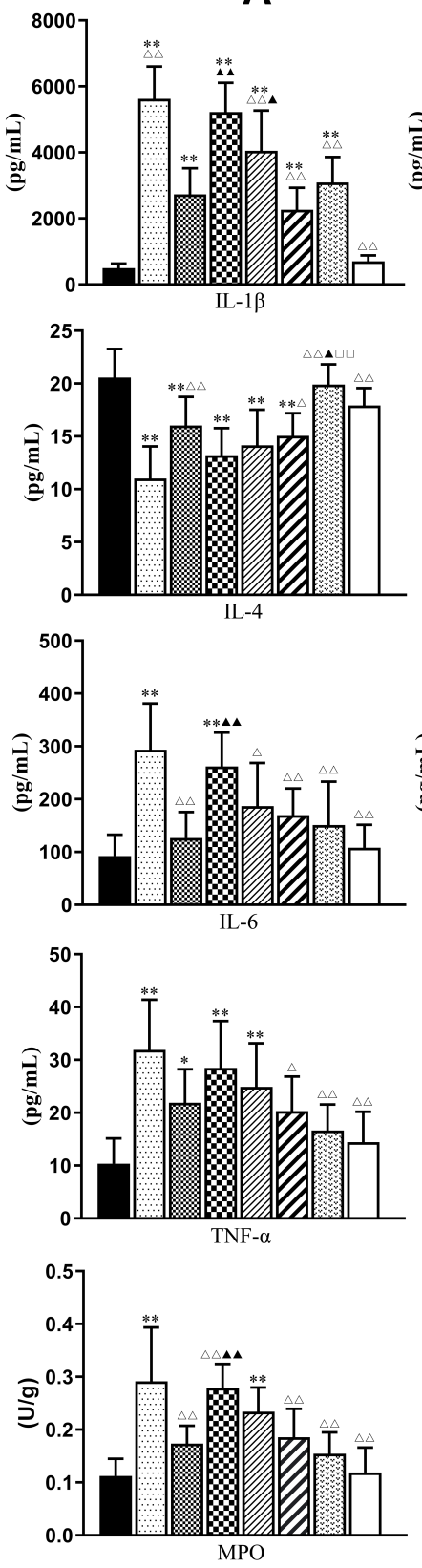

B
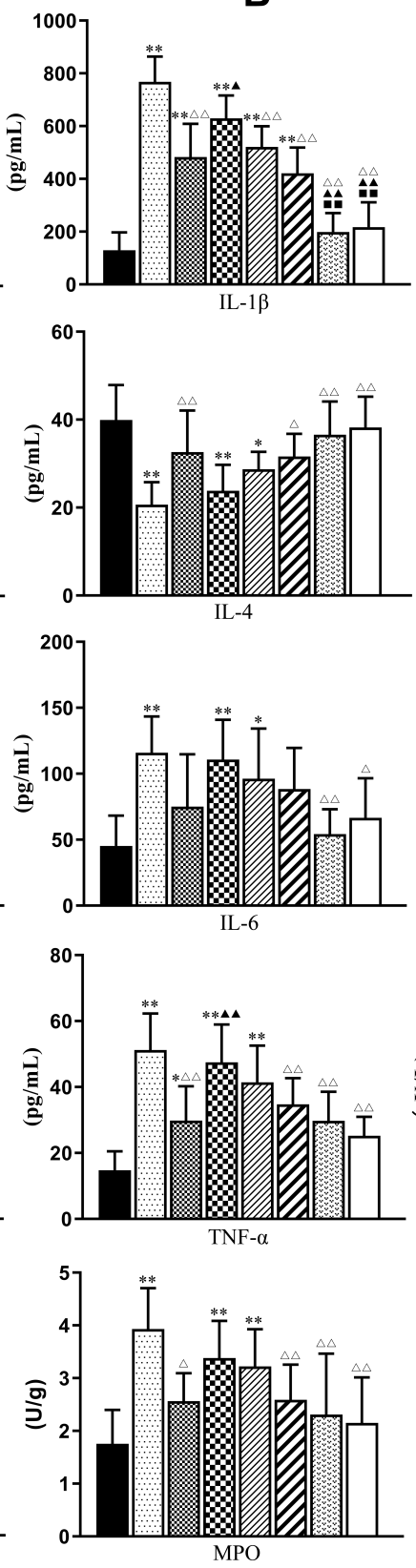

C
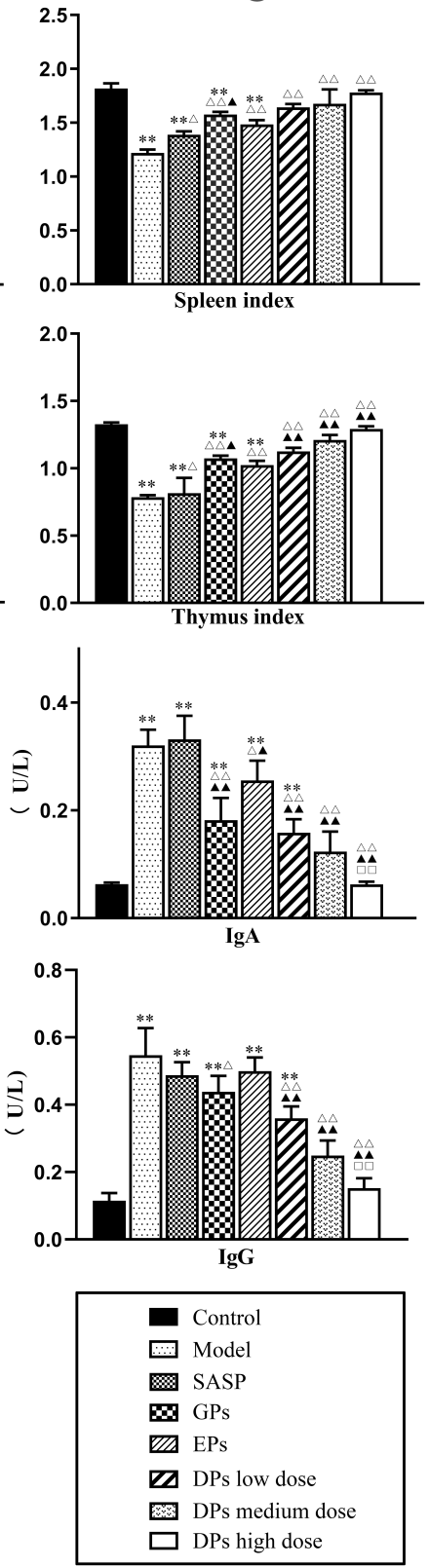

Figure 10 The effects of each treatment on the expression of inflammatory factors and immune-related indicators $(\bar{x} \pm s, \mathrm{n}=10) .(\mathbf{A})$ Serum, $(\mathbf{B})$ colon tissue, (C) serum. Comparisons: $* P<0.05,{ }^{* * P} P 0.01$ vs control group, ${ }^{\Delta} P<0.05,{ }^{\Delta \Delta} P<0.01$ vs model group, ${ }^{\Delta} P<0.05,{ }^{\Delta \Delta} P<0.01$ vs SASP group, ${ }^{\square} P<0.01$ vs $D P s$ low-dose group, ${ }^{-} P<0.05$ vs $D P s$ medium-dose group.

treatment with high-dose DPs. In the serum, except for the GPs group, each administration group showed a significant difference compared with the model group $(P<0.01)$, indicating that the effect of drug intervention was better than the effect of autologous recovery. The treatment with DPs in the medium/high-dose groups had significant effects on increasing the expression of IL-4 in serum and tissues $(P<0.01)$, and the promotive effect of IL-4 expression in the medium-dose group was more obvious. The control group was only had significantly different from the model group, the EPs group and GPs groups $(P<0.05)$, while the expression of IL-6 was clearly inhibited in the other groups. The expression level of IL-6 was more significantly decreased in the DPs medium/high-dose groups $(P<0.01)$. In addition, the expression level of TNF- $\alpha$ in the DPs groups was not significantly different from that in the control group $(P>0.05)$ in either serum or tissue, indicating that the DPs had a great effect on inhibiting the 
expression of TNF- $\alpha$. Additionally, there was a significant difference between the DPs medium dose, DPs high dose, SASP and model groups $(P<0.01)$, indicating that the activity of MPO was effectively inhibited in all three groups. All the results showed that DPs have great antiinflammatory effects, and the activity was even better than that of the positive control drug SASP and administration of EPs alone.

Compared with the control group, the spleen index and thymus index of the model rats were decreased, and the IgA and IgG levels in the serum were increased. Compared with the model group, the spleen index and thymus index of the rats in each DPs dose group were increased in a dose-dependent manner, and the $\operatorname{IgA}$ and IgG levels in the serum were also decreased in a dosedependent manner.

In the present study, after treatment with $\mathrm{AM}$ volatile oil-loaded GPs, the expression levels of IgA and IgG were obviously decreased. However, the levels were still significantly different from those in the healthy control group. To our satisfaction, the $\operatorname{IgA}$ and $\operatorname{IgG}$ concentrations in the DPs groups were decreased significantly. In particular, there was no significant difference between the DPs medium/high-dose groups and the control group $(P>0.05)$, suggesting that DPs can improve the immune function of rats with UC.

\section{Discussion}

Ulcerative colitis (UC) is a common autoimmune disease and is difficult to heal, or even lead to colon cancer in severe cases. At present, the clinical treatment of UC is still dominated by drug methods, while the commonly used chemotherapeutic drugs have some disadvantages that can only alleviate symptoms, and it is difficult to cure and the adverse reactions are obvious, which reduces the patient's compliance. Traditional Chinese medicine has unique advantages in the treatment of chronic gastrointestinal diseases caused by multiple reasons. It also pays attention to the overall balance while symptomatic treatment. In this study, two traditional Chinese medicines $\mathrm{BBR}$ and $\mathrm{AM}$ volatile oil were combined with an oral colon-specific drug delivery system, and the research was carried out on the preparation and in vitro-in vivo evaluation of DPs. Our results demonstrated that oral administration of DPs could effectively relieve the symptoms in UC rats' model, and the anti-UC mechanism may be related not only to inhibiting inflammation but also to enhancing body immunity.
In recent decades, state of the art small-animal imaging modalities provide non-invasive images rich in quantitative anatomical and functional information, which renders longitudinal studies possible allowing precise monitoring of disease progression and response to therapy in models of different diseases. ${ }^{43}$ Our research is significant because it offers the possibility of using in vivo imaging to position the delivery of oral preparation. Photographs were taken at regular intervals to observe transport and drug release from the pellets in the gastrointestinal tract. According to our vision, if the coating of the pellet is not destroyed, it should emit dot-like or flaky-like fluorescence. After the coating was dissolved, the punctate fluorescence gradually spread throughout the gastrointestinal tract with the release of the drug. Combined with the fluorescence signal and the anatomical features of the gastrointestinal tract of mice, the transport position of the EPs in the gastrointestinal tract can be judged to evaluate their in vivo targeting characteristics. To our satisfaction, in vivo targeting evaluation results showed that the EPs have good colon targeting properties and can be transported to the colon to release drugs after oral administration, thereby promoting drug absorption and improving drug efficacy.

MPO is an index of neutrophil recruitment in the murine UC model. Therefore, the MPO activity may reflect more specific inflammatory events compared with cytokine concentrations. In our study, the colonic MPO activity was significantly increased in the DSS group compared with the normal control group, and the group received with DPs showed significantly $(P<0.01)$ decreased MPO activity (Figure 10). Accordingly, DPs blocks neutrophil infiltration into injured tissues. Cytokines are considered crucial signals in the intestinal immune system, and immune cells, such as, T cells, dendritic cells, macrophages, and intestinal epithelial cells, are involved in the secretion of various cytokines that regulate the inflammatory response in UC. Previous studies have revealed elevated levels of cytokines, such as TNF- $\alpha$, IFN$\gamma$, IL-1 $\beta$, IL-6, IL-17, and IL- 21, in UC. ${ }^{30,44,45}$ In our study, we detected increased expression levels of TNF- $\alpha$, IL-1 $\beta$ and IL-6 in DSS-induced mice. However, the oral administration of DPs resulted in decreased expression levels of the above-mentioned proinflammatory cytokine.

The spleen and thymus are important immune organs in the body. It has been reported that the spleen index and thymus index of rats with UC are significantly reduced, and the immune capacity of organs is reduced. ${ }^{46}$ Immunoglobulins participate in humoral immunity, and 
the increased levels of $\operatorname{IgA}$ and $\operatorname{IgG}$ suggest that the humoral immunity of rats with UC is hyperactive, which may be a compensatory effect in response to the reduced cellular immunity. Studies have shown that an imbalance in the secretion of anti-inflammatory and pro-inflammatory factors is an important link in the intestinal inflammatory lesions of patients with UC. An increase in the levels of pro-inflammatory factors is involved in cellular immunity and promotes intestinal inflammatory responses, which can cause further damage to intestinal tissues. In our research, the expression levels of pro-inflammatory factors include TNF- $\alpha$, IL- $1 \beta$ and IL-6 in DPs received rats' groups were significantly decreased, and the expression level of anti-inflammatory factors such as IL-4 was relatively increased. In addition, the IgA and IgG concentrations in the DPs groups were significantly decreased. In summary, the orally deliverable DPs have a synergistic treatment effects for UC. It can regulate body immunity while exerting anti-inflammatory effects. The research of this subject is expected to provide a new drug for the treatment of UC.

\section{Conclusions}

A promising dual-targeted pellet loaded with BBR and AM volatile oil was developed for the treatment of UC. BBR was loaded in the enteric soluble layer for colontargeted release and thus had a local effect. AM volatile oil loaded in stomach-targeted pellets was prepared by extrusion-spheronization and fluid bed techniques, with HPMC as the gastric soluble layer, resulting in quick release and a systemic effect. The optimized EPs and GPs both have satisfying physicochemical characteristics. The bioavailability of BBR and AT-1 was much higher after pelletizing. The in vivo targeting evaluation showed that EPs have great acid resistance and satisfactory colon-targeting ability. The GPs disintegrated in $5 \mathrm{~min}$ in the stomach, allowing complete drug release. The synergistic effect of DPs ameliorated the clinical symptoms of UC, inhibited the expression of several inflammatory cytokines and decreased the levels of IgA and IgG. This suggests that the anti-UC mechanism may be related not only to inhibiting inflammation but also to enhancing body immunity. Thus, the developed oral dual-targeted delivery system could substantially contribute to the management of UC and might provide a pharmaceutical strategy for the synergistic treatment of gastrointestinal tract diseases.

\section{Acknowledgments}

The work was supported by the projects of the National Natural Science Foundation of China (81873011, 82074272), the Science and Technology Commission of Shanghai Municipality (20S21900300, 21XD1403400), the Outstanding Talents Program of Shanghai Health and Family Planning Commission (2018BR27). The authors thank the professor Fuzheng Ren of Shanghai Key Laboratory of New Drug Design, School of Pharmacy, East China University of Science and Technology, Shanghai, China, for his help in using the extruded-spheronizing granulator and fluidized bed coating-machine for this study.

\section{Disclosure}

The authors declare no conflicts of interest in this work.

\section{References}

1. Abraham C, Cho JH. Inflammatory bowel disease. $N$ Engl J Med. 2009;361(21):2066-2078. doi:10.1056/NEJMra0804647

2. Sicilia B, Garcia-Lopez S, Gonzalez-Lama Y, Zabana Y, Hinojosa J, Gomollon F. GETECCU 2020 guidelines for the treatment of ulcerative colitis developed using the GRADE approach. Gastroenterol Hepatol. 2020;43(Suppl 1):1-57. doi:10.1016/j.gastrohep.2020.07.001

3. Gajendran M, Loganathan P, Jimenez G, et al. A comprehensive review and update on ulcerative colitis(). Dis Mon. 2019;65 (12):100851. doi:10.1016/j.disamonth.2019.02.004

4. Han W, Xie B, Li Y, Shi L, Wang H. Orally deliverable nanotherapeutics for the synergistic treatment of colitis-associated colorectal cancer. Theranostics. 2019;9(24):7458-7473. doi:10.7150/ thno. 38081

5. Grivennikov SI. Inflammation and colorectal cancer: colitis-associated neoplasia. Semin Immunopathol. 2013;35:229-244.

6. Danese S, Mantovani A. Inflammatory bowel disease and intestinal cancer: a paradigm of the Yin-Yang interplay between inflammation and cancer. Oncogene. 2010;29(23):3313-3323. doi:10.1038/ onc.2010.109

7. Porter RJ, Kalla R, Ho GT. Ulcerative colitis: recent advances in the understanding of disease pathogenesis. F1000Res. 2020;9:294. doi:10.12688/f1000research.20805.1

8. Wang R, Zhou G, Wang M, Peng Y, Li X. The metabolism of polysaccharide from Atractylodes macrocephala Koidz and its effect on intestinal microflora. Evid Based Compl Alt. 2014;2014:1-7.

9. Zhang L, Cao N, Wang Y, et al. Improvement of oxazolone-induced ulcerative colitis in rats using andrographolide. Molecules. 2020;25 (1):76. doi:10.3390/molecules25010076

10. Kringel DH, Antunes MD, Klein B, et al. Production, characterization, and stability of orange or eucalyptus essential oil/beta-cyclodextrin inclusion complex. J Food Sci. 2017;82(11):2598-2605. doi:10.1111/1750-3841.13923

11. Tan S, Yu W, Lin Z, et al. Berberine ameliorates intestinal mucosal barrier damage induced by peritoneal air exposure. Biol Pharm Bull. 2015;38(1):122-126. doi:10.1248/bpb.b14-00643

12. Li YH, Xiao H, Hu D, et al. Berberine ameliorates chronic relapsing dextran sulfate sodium-induced colitis in C57BL/6 mice by suppressing Th17 responses. Pharmacol Res. 2016;110:S1943242085.

13. Liao Z, Xie Y, Zhou B, et al. Berberine ameliorates colonic damage accompanied with the modulation of dysfunctional bacteria and functions in ulcerative colitis rats. Appl Microbiol Biotechnol. 2020;104 (4):1737-1749. doi:10.1007/s00253-019-10307-1 
14. Leopold CS, Eikeler D. Basic coating polymers for the colon-specific drug delivery in inflammatory bowel disease. Drug Dev Ind Pharm. 2000;26(12):1239-1246. doi:10.1081/DDC-100102305

15. Yang L, Chu JS, Fix JA. Colon-specific drug delivery: new approaches and in vitro/in vivo evaluation. Int $J$ Pharmaceut. 2002;235(1):1-15. doi:10.1016/S0378-5173(02)00004-2

16. Lee SH, Bajracharya R, Min JY, Han JW, Park BJ, Han HK. Strategic approaches for colon targeted drug delivery: an overview of recent advancements. Pharmaceutics. 2020;12(1):68.

17. Wang Q, Wang G, Zhou J, Gao L, Cui Y. Colon targeted oral drug delivery system based on alginate-chitosan microspheres loaded with icariin in the treatment of ulcerative colitis. Int $J$ Pharmaceut. 2016;515(1-2):176-185. doi:10.1016/j.ijpharm.2016.10.002

18. Xiao B, Merlin D. Oral colon-specific therapeutic approaches toward treatment of inflammatory bowel disease. Expert Opin Drug Del. 2012;9(11):1393-1407. doi:10.1517/17425247.2012.730517

19. Pandey S, Swamy S, Gupta A, et al. Multiple response optimisation of processing and formulation parameters of $\mathrm{pH}$ sensitive sustained release pellets of capecitabine for targeting colon. $J$ Microencapsul. 2018;35(3):259-271. doi:10.1080/02652048.2018.1465138

20. Sun $\mathrm{X}$, Liu $\mathrm{C}$, Omer $\mathrm{AM}$, et al. $\mathrm{pH}$-sensitive $\mathrm{ZnO} /$ carboxymethyl cellulose/chitosan bio-nanocomposite beads for colon-specific release of 5-fluorouracil. Int $J$ Biol Macromol. 2019;128:468-479. doi:10.1016/j.ijbiomac.2019.01.140

21. Moghimipour E, Rezaei M, Kouchak M, et al. Effects of coating layer and release medium on release profile from coated capsules with Eudragit FS 30D: an in vitro and in vivo study. Drug Dev Ind Pharm. 2018;44(5):861-867. doi:10.1080/03639045.2017.1415927

22. Wan D, Zhao M, Zhang J, Luan L. Development and in vitro-in vivo evaluation of a novel sustained-release loxoprofen pellet with double coating layer. Pharmaceutics. 2019;11(6):260. doi:10.3390/ pharmaceutics 11060260

23. Segale L, Mannina P, Giovannelli L, Muschert S, Pattarino F. Formulation and coating of alginate and alginate-hydroxypropylcellulose pellets containing ranolazine. $J$ Pharm Sci US. 2016;105(11):3351-3358. doi:10.1016/j.xphs.2016.08.001

24. Kim Y, Pradhan R, Paudel BK, Choi JY, Im HT, Kim JO. Preparation and evaluation of enteric-coated delayed-release pellets of duloxetine hydrochloride using a fluidized bed coater. Arch Pharm Res. 2015;38 (12):2163-2171. doi:10.1007/s12272-015-0590-y

25. Melegari C, Bertoni S, Genovesi A, et al. Ethylcellulose film coating of guaifenesin-loaded pellets: a comprehensive evaluation of the manufacturing process to prevent drug migration. Eur J Pharm Biopharm. 2016;100:15-26. doi:10.1016/j.ejpb.2015.12.001

26. Belew S, Suleman S, Duguma M, et al. Development of a dissolution method for lumefantrine and artemether in immediate release fixed dose artemether/lumefantrine tablets. Malar J. 2020;19(1):139. doi:10.1186/s12936-020-03209-5

27. Verstraete G, De Jaeghere W, Vercruysse J, et al. The use of partially hydrolysed polyvinyl alcohol for the production of high drug-loaded sustained release pellets via extrusion-spheronisation and coating: in vitro and in vivo evaluation. Int $J$ Pharmaceut. 2017;517(12):88-95. doi:10.1016/j.ijpharm.2016.11.067

28. Novobilsky A, Hoglund J. Small animal in vivo imaging of parasitic infections: a systematic review. Exp Parasitol. 2020;214:107905. doi:10.1016/j.exppara.2020.107905

29. Karrout Y, Dubuquoy L, Piveteau C, et al. In vivo efficacy of microbiota-sensitive coatings for colon targeting: a promising tool for IBD therapy. J Control Release. 2015;197:121-130. doi:10.1016/ j.jconrel.2014.11.006

30. Pandurangan AK, Ismail S, Saadatdoust Z, Esa NM. Allicin alleviates dextran sodium sulfate- (DSS-) induced ulcerative colitis in BALB/c mice. Oxid Med Cell Longev. 2015;2015:605208. doi: $10.1155 / 2015 / 605208$
31. Ferri D, Costero AM, Gavina P, et al. Efficacy of budesonide-loaded mesoporous silica microparticles capped with a bulky azo derivative in rats with TNBS-induced colitis. Int J Pharm. 2019;561:93-101. doi:10.1016/j.ijpharm.2019.02.030

32. Shi C, Liang Y, Yang J, et al. MicroRNA-21 knockout improve the survival rate in DSS induced fatal colitis through protecting against inflammation and tissue injury. Plos One. 2013;8(6):e66814. doi:10.1371/journal.pone.0066814

33. Nunes NS, Chandran P, Sundby M, et al. Therapeutic ultrasound attenuates DSS-induced colitis through the cholinergic anti-inflammatory pathway. Ebiomedicine. 2019;45:495-510. doi:10.1016/j. ebiom.2019.06.033

34. Li X, Xu Y, Zhang C, et al. Protective Effect of Calculus Bovis Sativus on Dextran Sulphate Sodium-Induced Ulcerative Colitis in Mice. Evid-Based Compl Alt. 2015;2015:Article ID 469506. doi: $10.1155 / 2015 / 469506$

35. Gao W, Wang C, Yu L, et al. Chlorogenic Acid Attenuates Dextran Sodium Sulfate-Induced Ulcerative Colitis in Mice through MAPK/ ERK/JNK Pathway. Biomed Res Int. 2019;2019:Article ID: 6769789. doi: $10.1155 / 2019 / 6769789$

36. Ke J, Bian X, Liu H, et al. Edaravone reduces oxidative stress and intestinal cell apoptosis after burn through up-regulating miR-320 expression. Mol Med. 2019;25:54. doi:10.1186/s10020-019-0122-1

37. Kverka M, Rossmann P, Tlaskalova-Hogenova H, et al. Safety and efficacy of the immunosuppressive agent 6-tioguanine in murine model of acute and chronic colitis. Bmc Gastroenterol. 2011;11 (1):47. doi:10.1186/1471-230X-11-47

38. Banerjee A, Bizzaro D, Burra P, et al. Umbilical cord mesenchymal stem cells modulate dextran sulfate sodium induced acute colitis in immunodeficient mice. Stem Cell Res Ther. 2015;6:79. doi:10.1186/ s13287-015-0073-6

39. Neurath MF. Cytokines in inflammatory bowel disease. Nat Rev Immunol. 2014;14(5):329-342. doi:10.1038/nri3661

40. Kramar A, Turk S, Vrečer F. Statistical optimisation of diclofenac sustained release pellets coated with polymethacrylic films. Int $J$ Pharmaceut. 2003;256(1-2):43-52. doi:10.1016/S03785173(03)00061-9

41. Wang X, Luo Z, Xiao Z. Preparation, characterization, and thermal stability of $\beta$-cyclodextrin/soybean lecithin inclusion complex. Carbohyd Polym. 2014;101:1027-1032. doi:10.1016/j. carbpol.2013.10.042

42. Kumar A, Ekavali CK, Mukherjee M, Pottabathini R, Dhull DK, Dhull DK. Current knowledge and pharmacological profile of berberine: an update. Eur J Pharmacol. 2015;761:288-297. doi:10.1016/ j.ejphar.2015.05.068

43. Lauber DT, Fulop A, Kovacs T, Szigeti K, Mathe D, Szijarto A. State of the art in vivo imaging techniques for laboratory animals. $L a b$ Anim. 2017;51(5):465-478. doi:10.1177/0023677217695852

44. Araki A, Nara H, Rahman M, et al. Role of interleukin-21 isoform in dextran sulfate sodium (DSS)-induced colitis. Cytokine. 2013;62 (2):262-271. doi:10.1016/j.cyto.2013.03.006

45. Pervin M, Hasnat MA, Lim JH, et al. Preventive and therapeutic effects of blueberry (Vaccinium corymbosum) extract against DSS-induced ulcerative colitis by regulation of antioxidant and inflammatory mediators. $J$ Nutr Biochem. 2016;28:103-113. doi:10.1016/j.jnutbio.2015.10.006

46. Gong Y, Niu W, Tang Y, et al. Aggravated mucosal and immune damage in a mouse model of ulcerative colitis with stress. Exp Ther Med. 2019;17(3):2341-2348. 


\section{Publish your work in this journal}

Drug Design, Development and Therapy is an international, peerreviewed open-access journal that spans the spectrum of drug design and development through to clinical applications. Clinical outcomes, patient safety, and programs for the development and effective, safe, and sustained use of medicines are a feature of the journal, which has also been accepted for indexing on PubMed Central. The manuscript management system is completely online and includes a very quick and fair peer-review system, which is all easy to use. Visit http://www. dovepress.com/testimonials.php to read real quotes from published authors.

Submit your manuscript here: https://www.dovepress.com/drug-design-development-and-therapy-journal 